Article

\title{
Liquid-Liquid Flow Pattern Prediction Using Relevant Dimensionless Parameter Groups
}

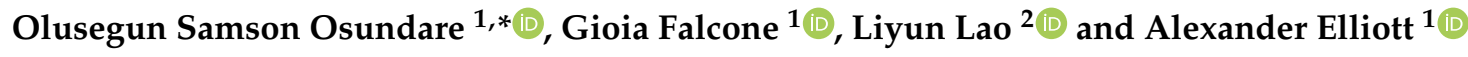 \\ 1 James Watt School of Engineering, University of Glasgow, Glasgow G12 8QQ, UK; \\ Gioia.Falcone@glasgow.ac.uk (G.F.); Alexander.Elliott@glasgow.ac.uk (A.E.) \\ 2 Centre for Thermal Energy Systems and Materials, Cranfield University, Bedford MK43 0AL, UK; \\ 1.lao@cranfield.ac.uk \\ * Correspondence: o.osundare.1@research.gla.ac.uk; Tel.: +44-7466878853
}

Received: 15 June 2020; Accepted: 17 August 2020; Published: 24 August 2020

\begin{abstract}
Accurate predictions of flow patterns in liquid-liquid flow are critical to the successful design and operation of industrial and geo-energy systems where two liquids are jointly transported. Unfortunately, there is no unified flow pattern map, because all published maps are based on limited ranges of dimensional parameters. Dimensional analysis was performed on oil-water horizontal flows, to obtain some relevant dimensionless parameter groups (DPG) for constructing flow pattern maps (FPM). The following combinations of DPG were used: (i) the ratio of mixture Reynolds number to Eötvös number versus water fraction, (ii) the ratio of Weber number to Eötvös number versus water fraction, (iii) the mixture Froude number versus water fraction, (iv) the water Froude number versus oil Froude number, (v) the ratio of gravity force to viscous force versus water fraction. From twelve published experimental studies, 2696 data points were gathered and analysed covering a variety of flow patterns including stratified, stratified mixed, dispersed oil in water, dispersed water in oil, annular and slug flows. Based on the performed analysis, it was found that flow patterns could occupy more than one isolated region on the DPG-based flow pattern map. None of the combinations of DPG can mark out all the considered flow patterns, however, some combinations of DPG are particularly suitable for marking out the regions associated with some flow patterns.
\end{abstract}

Keywords: oil-water; flow regime; geo-energy; dimensionless numbers; oil-viscosity; pipe diameter

\section{Introduction}

Due to their applicability in various process industries, horizontal liquid-liquid flows have been extensively studied. Such research is particularly important in the energy sector, where oil and water are usually co-produced and transported jointly. However, despite the importance of liquid-liquid flows, they have not been studied to the same extent of the gas-liquid flows [1,2] and there is no unified flow pattern map for the liquid-liquid flows [3]. In most cases, the flow pattern maps available for the liquid-liquid flow are limited to the conditions for which the flow maps were constructed [4]. Figure 1 presents a liquid-liquid flow pattern map developed for mineral oil and tap water, featuring superficial phase velocities. Various other parameters have been employed in the construction of flow pattern maps, as discussed in Section 2.2. The use of dimensionless groups to develop flow pattern maps would lead to a wider range of applicability [5]. Therefore, this project focuses on the construction of flow pattern maps using various combinations of relevant dimensionless parameter groups to ensure wider usage in horizontal pipes.

The earliest research into liquid-liquid flow targeted the improvement of transportation of crude oil in pipelines [6-9], but liquid-liquid flow research is also important for flow in oil well production tubing, enhanced oil recovery (EOR) methods, and the design of chemical reactors and separators [10]. 


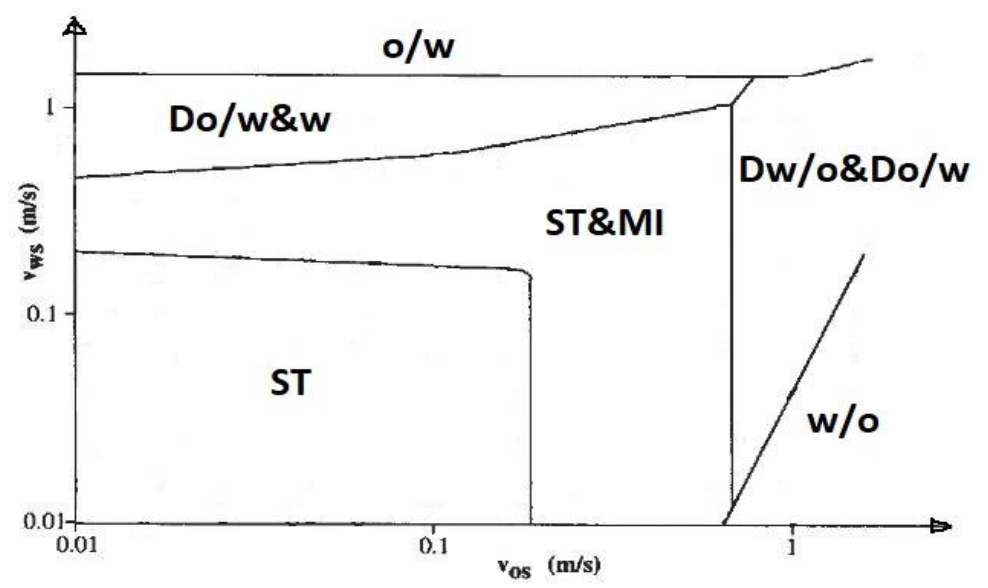

Figure 1. Graph of superficial water velocity versus superficial oil velocity modified after Trallero et al. [11] developed for an inner pipe diameter of $50.1 \mathrm{~mm}$, with $\mu_{\mathrm{o}}=0.0288 \mathrm{~Pa} \mathrm{~s}, \mu_{\mathrm{W}}=0.00097 \mathrm{~Pa} \mathrm{~s}, \rho_{\mathrm{o}}=884 \mathrm{~kg} / \mathrm{m}^{3}$, $\rho_{\mathrm{W}}=997^{*} \mathrm{~kg} / \mathrm{m}^{3}$ and $\sigma=0.036 \mathrm{~N} / \mathrm{m}$ at $25.5^{\circ} \mathrm{C}$. Note: for data with ${ }^{(*)}$, the authors recorded $1037 \mathrm{~kg} / \mathrm{m}^{3}$ for tap water at $\approx 25.5^{\circ} \mathrm{C}$.

\subsection{Importance of Flow Patterns in the Industrial Sector}

Understanding the nature and behaviour of liquid-liquid flow is critically important but also challenging, as many physical and, sometimes, chemical mechanisms govern the formation of flow patterns. Once a flow pattern is determined, some key process parameters, such as pressure gradients and phase fractions of that particular flow system could be estimated.

In oil-water pipelines, stratified flow exists at low velocities with oil flowing at the top while water flows at the bottom in a horizontal pipe. The bottom section of the pipeline is prone to corrosion due to the continuous flow of water $[12,13]$ and the dissolution of gases, such as $\mathrm{CO}_{2}$ and $\mathrm{H}_{2} \mathrm{~S}$ in water, which are attributed as the cause of corrosion. The oil phase by itself is considered non-corrosive [14], so in theory, a pipeline which has no free-flowing water phase (i.e., the water phase is entirely entrained in the oil phase) is free of corrosion problems [13,15].

It is essential to determine the flow pattern for an optimal process of injecting inhibitors and other chemicals into the pipeline to prevent corrosion. Furthermore, predicting the flow pattern of a pipeline is essential for calculating the pressure drop along it, which improves design and optimises maintenance of the pipe itself.

At ambient conditions, viscous heavy oil does not flow easily. Before transporting such crude, its high viscosity is usually reduced, by either heating it, adding a diluent, or both. Charles et al. [7] and Shi et al. [16] found that the introduction of water into a heavy oil pipeline can reduce both the pressure gradient along it and the power required to pump a given amount of the crude. An ideal flow pattern for transporting heavy oil is the core-annular flow (CAF), where the crude flows in the core, while a small quantity/amount of water forms annulus around the oil and lubricates the pipeline $[2,7,10,16]$. The injected water is pre-treated with a demulsifying agent to help the phase separation at the end of the pipeline [17], so flow pattern determination can suggest the most appropriate way of transporting multiphase flow fluids.

There is a substantial difference in the pressure gradients encountered with various flow patterns [17]. For dispersed flows, a system of two immiscible fluids (oil and water) can become more complex as the resulting mixture fluid may become an emulsion, which is unstable when it separates into the original phases that formed it within a reasonable period of time at rest.

When designing an oil-water pipeline, an accurate prediction of the phase inversion point is desirable as there is a significant difference between the pressure drop in the oil-dominated and the water-dominated regions [17]. Phase inversion is a phenomenon in oil-water flow systems where the dispersed phase switches to be the continuous phase. With saline water, the inversion point can be observed earlier than with tap water ( 0.45 and 0.5 oil fraction for saline and tap water, respectively) [18]. 
At the inversion point, the oil-water mixture viscosity can increase dramatically, so pipeline operators strive to avoid operating at this flow condition [17]. This can be achieved with a good understanding of the system flow patterns.

\subsection{Liquid-Liquid Flow Patterns in Horizontal Pipes}

When two immiscible liquids flow together in a horizontal pipeline, different flow patterns are experienced. The factors responsible for the flow pattern configurations include density ratio, viscosity ratio, input phase ratio, mixture flow rate, wetting properties, surface tension and pipe geometry $[2,19]$. For example, using the same experimental facility, two different flow pattern descriptions were obtained by two researchers, who operated at the same conditions, but used two various working fluids [11]. The name given to a particular flow pattern may not always be meaningful as it does not describe the features of the flow pattern [11]. Various researchers have described the flow patterns with different names and terminologies, Section 2.3 resolves this challenge. In addition, the number of flow patterns reported for liquid-liquid horizontal pipes varies from one researcher to another with Oglesby [20], Trallero et al. [11], Nädler and Mewes [21], Brauner [2] and Al-Wahaibi et al. [22] reporting fourteen, six, seven, eighteen and six flow patterns, respectively. Figures $2-4$ present some of these flow patterns.
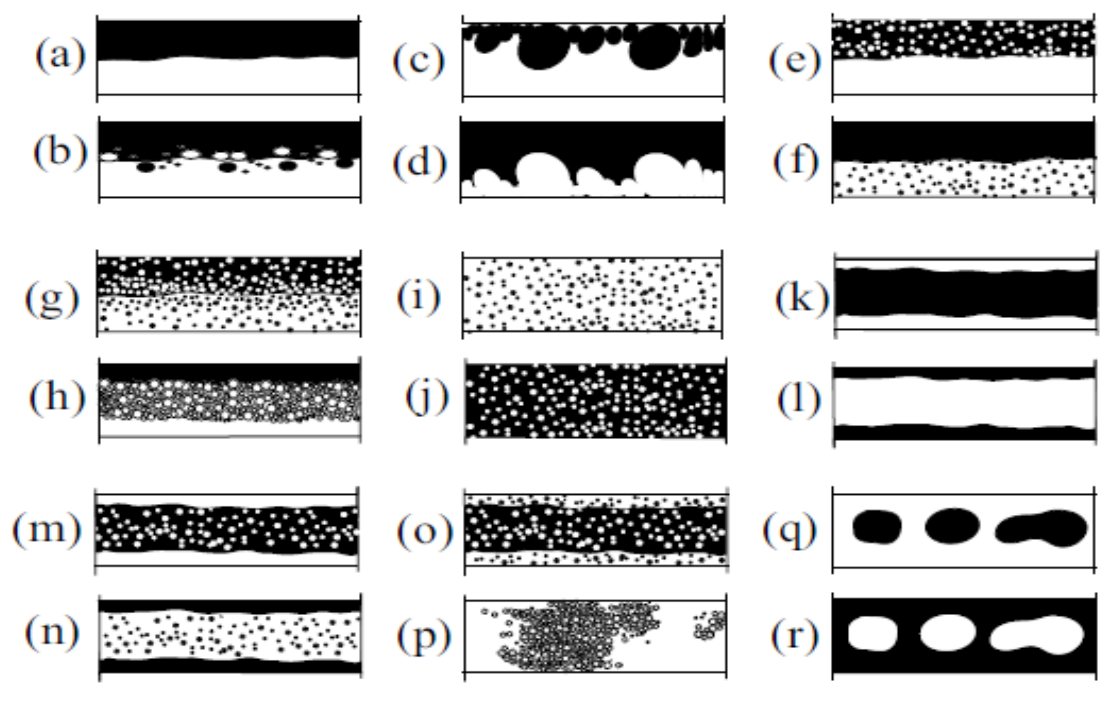

\section{(i)}

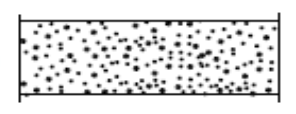

(j)

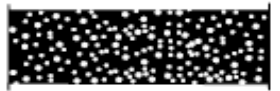

(k)

(1)

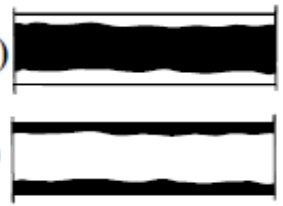

(o)

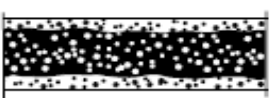

(p)
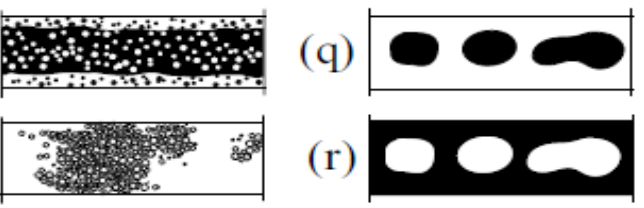

Figure 2. Typical flow patterns of oil-water in horizontal pipes [2]. Note: $(\mathbf{a}, \mathbf{b})$ Stratified flow of two separated layers (S, possibly with mixing at the interface, SM). (c,d) Stratified layers of a free-liquid and a dispersion of the other liquid (e.g., oil-in-water dispersion above a water layer, Do/w \& w). $(\mathbf{e}, \mathbf{f})$ Stratified layers of a free liquid and a dispersion in the other liquid (e.g., oil and oil-in-water dispersion, Do/w \& o; water and water-in-oil dispersion, Dw/o \& w). (g,h) Layer of dispersions (e.g., water-in-oil dispersion above oil-in-water dispersion Dw/o \& o/w, possibly with pure oil at the top and/or water at the bottom). (i,j) Fully dispersion or emulsion of one liquid in the other liquid (e.g., water-in-oil or oil-in-water dispersion or emulsion, Dw/o or Do/w). (k,1) Core-annular flow-a core of one liquid with the other liquid (e.g., a core of viscous oil and water in the annulus, ANw. Oil in the annulus, ANo). (m,n) Annular flow of a liquid with a dispersion in the core (water in the annulus DANw, oil in the annulus DANo). (o) Core-annular flow of two dispersions (CADw or CADo). (p) Intermittent flow (one liquid alternatively occupying the pipe as free liquid or as a dispersion, Io or Iw). (q,r) Larger elongated or spherical bubbles of one liquid in the other (SLo, Bo or SLw, Bw) [2]. 
Stratified Flow (ST)

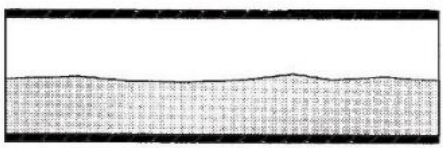

Dispersion of oil in water and water (Do/w \& w)

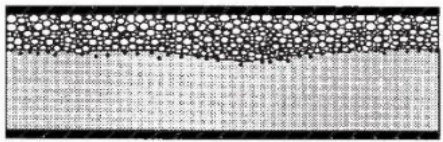

Dispersions of water in oil and oil in water (Dw/o \& Do/w )

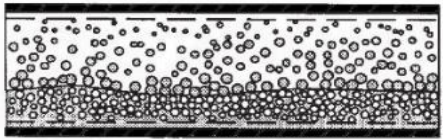

Stratified Flow with mixing at the interface (ST \& MI)

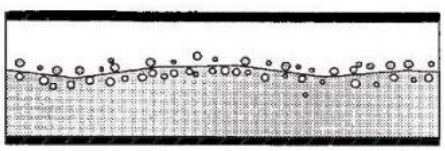

Oil in water emulsion $(0 / w)$

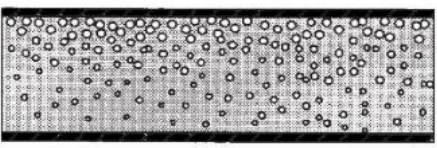

Water in oil emulsion (w/o)

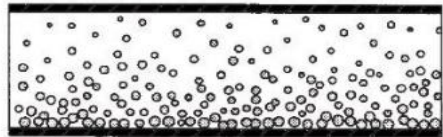

Figure 3. Typical horizontal oil-water flow pattern sketches modified after Trallero et al. [11].

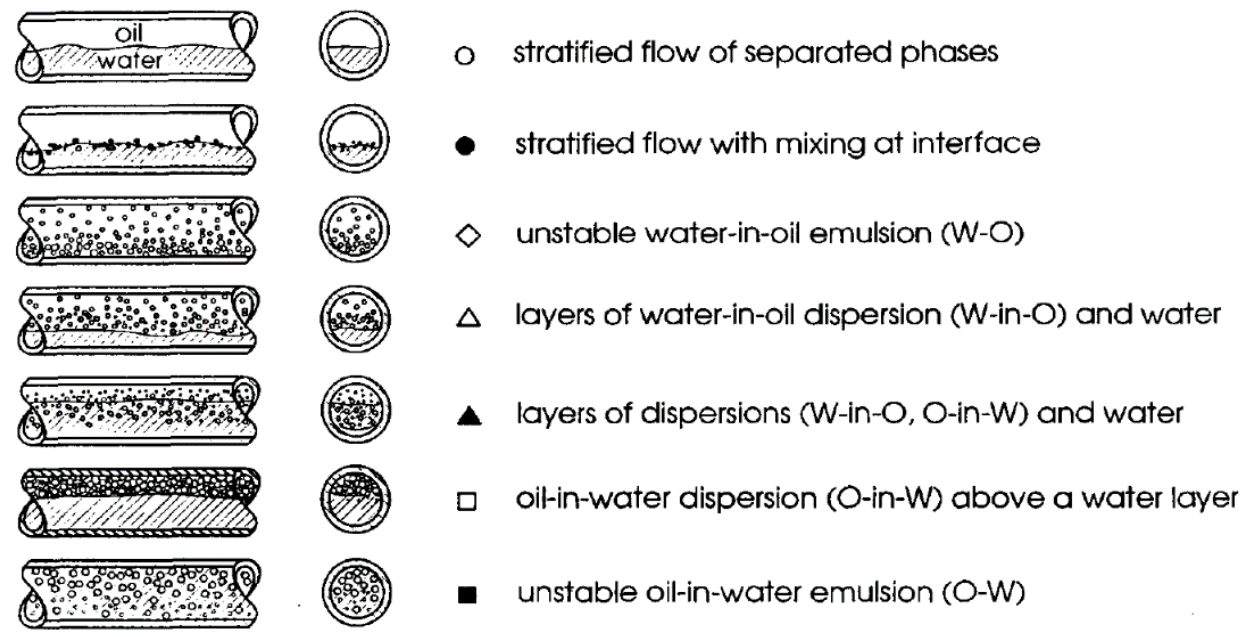

Figure 4. Schematic diagram of oil-water flow pattern in a horizontal pipeline [21].

For an accurate prediction of the multiphase flow features, it is essential to identify the flow pattern, because uncertainties in flow pattern determination greatly affect the liquid-hold up and pressure drop predictions [23]. Therefore, the flow pattern is a defining feature of multiphase flow. In predicting local flow pattern, flow pattern maps are employed. A flow pattern map is generally a 2-D graph with transition boundaries which separate the graph into areas with similar flow configuration called flow pattern [24].

A literature review of two immiscible liquids flowing in the horizontal pipeline showed that there is no unified/standard flow pattern map for all investigators [3]. Different investigators have presented their flow pattern maps on various coordinates which makes the comparison and interpretation of the published flow patterns difficult [11]. A detailed discussion of the chronological development of flow pattern maps in Section 2.2. The application of flow pattern maps based on dimensional parameters is limited to the particular pipe size and fluids used for constructing them [4]. Flow pattern maps with a wider range of applicability can be achieved when relevant dimensionless parameters are used for constructing them [5]. 


\section{Data Collection}

The key aim of this study is to utilise relevant dimensionless parameters to develop more accurate flow pattern maps for liquid-liquid flow in horizontal pipes. As such, an important initial step is a thorough investigation into the relevant literature to scope suitable experimental studies and to create a pool of data points to allow meaningful trends to be observed. Theoretical studies have also been considered in this process as, although they do not contribute to the flow maps themselves, they provide valuable insight into the physical phenomena and allow a more informed set of flow maps to be developed.

As there are a number of geometric, flow conditions and fluid characteristics that may influence the flow pattern $[19,25]$, a rigorous approach has been taken to ensure consistency between the experimental studies included in this study. The selected experimental studies were based largely on datasets accessibility, pipe inclination and flow conditions. The main criteria for inclusion are presented in Table 1.

This section provides an overview of the considered literature and also highlights the reasons for the inclusion of the experimental studies in the final data set. It should be noted that all reasonable efforts were made to acquire the experimental flow maps, but that this was not always possible if the data had not been presented or made available for download.

Finally, it can be noted that, while the intention of this section was not to create a literature review, sufficient detail has been included to provide a thorough overview of research in this area.

Table 1. Main conditions for including an experimental study.

\begin{tabular}{|c|c|c|c|}
\hline Group of Factors & Factor & Criterion & Reasons \\
\hline \multirow[b]{2}{*}{ Pipe Geometry } & Pipe inclination & Horizontal $\left(0^{\circ}\right)$ & $\begin{array}{l}\text { This study focuses solely on horizontal flow, as pipe inclination } \\
\text { influences the interaction between the dominant forces (inertial, } \\
\text { gravity and buoyancy), which can delay or accelerate the } \\
\text { emergence of a flow pattern [26] }\end{array}$ \\
\hline & Pipe internal diameter & $10-100 \mathrm{~mm}$ & $\begin{array}{l}\text { - So far most of the studies of multiphase pipe flows were } \\
\text { conducted in the closed channels with a hydraulic diameter } \\
\text { ranged from } 10 \mathrm{~mm} \text { to } 100 \mathrm{~mm} \text {. The multiphase flow behaviors in } \\
\text { small diameter }(<10 \mathrm{~mm} \text { ) pipes are significantly different from } \\
\text { that in the pipes of conventional scales, as the Eötvös numbers in } \\
\text { the small pipes are small hence the interfacial tension is critically } \\
\text { significant in comparing to the gravitational forces. On the other } \\
\text { hand, Cheng et al. [27] also showed that behaviors of multiphase } \\
\text { flows in large diameter ( }>100 \mathrm{~mm}) \text { pipes could be substantially } \\
\text { different from that in smaller pipes }\end{array}$ \\
\hline \multirow{3}{*}{ Fluid Properties } & Density & $\begin{array}{l}\text { Water }\left(\sim 1000 \mathrm{~kg} / \mathrm{m}^{3}\right), \text { oil } \\
\quad\left(750-900 \mathrm{~kg} / \mathrm{m}^{3}\right)\end{array}$ & $\begin{array}{l}\text { - The buoyancy and gravity forces are direct function of density [26] } \\
\text { - At relatively low flow rate, high density difference between the } \\
\text { two phases results in stratified flow [28-30] } \\
\text { - With equal density phases, no stratification [7] }\end{array}$ \\
\hline & Viscosity & $\begin{array}{l}\text { Water }(0.8-1.0 \mathrm{mPa} s), \text { oil } \\
\quad(<700 \mathrm{mPa} \mathrm{s})\end{array}$ & $\begin{array}{l}\text { - Interfacial instability increases as the difference in viscosity } \\
\text { between the two phases increases [28] }\end{array}$ \\
\hline & Interfacial tension & $0.01-0.06 \mathrm{~N} / \mathrm{m}$ & $\begin{array}{l}\text { - From moderate to relatively high velocities, interfacial instability } \\
\text { is high due to high turbulence, which leads to faster transition } \\
\text { from stratified to non-stratified flow [28] }\end{array}$ \\
\hline \multirow[b]{2}{*}{ Flow Conditions } & Temperature & $\sim 25^{\circ} \mathrm{C}$ & $\begin{array}{l}\text { - The operating temperature of an experimental set-up has a } \\
\text { significant influence on the physical properties of the fluids [31] }\end{array}$ \\
\hline & Pressure & $\sim 1 \mathrm{~atm}$ & $\begin{array}{l}\text { - Oil and water are usually treated as incompressible fluids, hence } \\
\text { the pressure effect on physical properties of liquid-liquid flow is } \\
\text { considered negligible [32] }\end{array}$ \\
\hline
\end{tabular}

\subsection{Experimental Studies on Liquid-Liquid Flow in Horizontal Pipelines}

A summary of some relevant experimental works published on liquid-liquid flow in horizontal pipes is provided in Table 2. The pipe diameters vary from $5.6 \mathrm{~mm}$ to $101.2 \mathrm{~mm}$, with the majority 
ranging from $19 \mathrm{~mm}$ to $30 \mathrm{~mm}$, and just two experimental works having pipe diameters less than $19 \mathrm{~mm}[33,34]$. Only a few of the experimental studies used pipe diameters greater than $60 \mathrm{~mm}$ [35-37]. The ratios of oil to water viscosity and density range from $0.7 \leq \mu_{\mathrm{o}} / \mu_{\mathrm{w}} \leq 12550$, and $0.684 \leq \rho_{\mathrm{o}} / \rho_{\mathrm{w}} \leq 1$, respectively, whilst the interfacial tension $(\sigma)$ ranges from $0.017 \mathrm{~N} / \mathrm{m}$ to $0.05 \mathrm{~N} / \mathrm{m}$.

Determination of flow pattern is the key to modelling of liquid-liquid flow as all of the design variables are dependent on the flow pattern, e.g., the pressure drop of oil-water flow in a horizontal pipeline depends on the flow pattern $[6,7,17,21,38,39]$. Experimental studies relevant to this project that have been performed to determine flow patterns in oil-water horizontal flow are presented chronologically in Table 2.

The data provides an idea about the pipe roughness and wettability, which influence the pressure gradient, flow patterns and the hold-up. Angeli and Hewitt [30] observed that flow patterns were more disturbed in steel pipes than in acrylic pipe, which explained why there was a very small stratified wavy region and the mixed region began at lesser velocities in the former.

Recent studies on liquid-liquid flow have focused more on high viscous oil-water flow due to its relevance to the petroleum industry activities enhanced oil recovery (EOR) and heavy oil transportation [40]. Annular flow, a flow pattern associated with the highly viscous oil-water flow system, is more pronounced in those studies where high viscosity oil is used [41-43]. Detailed studies of the annular flow pattern are necessary to reduce the head-loss due to high oil viscosity during transportation [40,44]. Charles et al. [7] suggested that high and low viscosity oils behave differently due to their wall wetting effects. Note that oil viscosity is classified into $<10 \mathrm{mPa} \mathrm{s}, 20-100 \mathrm{mPa}$ and $>100 \mathrm{mPa}$ s as low-viscous oil, medium-viscous oil and heavy-viscous oil, respectively $[3,45]$.

Where there is a small density difference $(\Delta \rho)$ between the two liquids for a liquid-liquid flow system, the effect of gravitational forces is reduced. The wetting ability and interfacial tension properties of liquids have a significant impact on the flow patterns $[10,15]$. When two immiscible liquids flow together in a pipe, they tend to stratify, but the higher the density difference $(\Delta \rho)$ is, the better is the achieved stratification [7].

Interfacial tension and the gravity forces tend to stabilise the interface between the layers of two immiscible liquids. Yet when these stabilising forces are less than the destabilising forces (relative movement and the viscosity difference between the two liquids, the interface becomes destabilised [22,28,46]. High interfacial tension between the two liquids will prevent the growth of amplitude at the interface and thereby stabilise the interface.

There have been significant developments in flow patterns detection over the years. The measuring techniques range from simple to more sophisticated techniques such as visual observation, photography (video-camera), high-frequency impedance probe, conductivity needle probe, particle image velocimetry, dual impedance probe, and hot-wire anemometry.

As discussed in Section 1.2, density ratio, viscosity ratio, input phase ratio, mixture flow rate, wetting properties, surface tension and pipe geometry are factors influencing the flow patterns [2].

Wang and Gong [47] showed the differences between high viscosity mineral oil-water flow and crude oil-water flow in horizontal pipelines, commenting that mineral oil was more widely used than the crude oil in the liquid-liquid flow experimental studies. Even where both mineral and crude oils had approximately the same viscosity and density, they found out that their flow patterns and transition boundaries did not agree. In terms of similarity, they observed that the flow patterns of both mineral oil-water and crude oil-water flow systems could be grouped into oil-dominated, water-dominated, intermittent, and stratified regions. The presence of some natural surfactants or depositions in crude oil, such as resin and asphaltenes caused a delay in the transition from one flow pattern to another [40,48].

The choice of mixing device is essential in the experimental studies, as each device promotes the formation of a particular flow pattern. An injecting device promotes core-annular flow [40] whereas cones or special " $\mathrm{Y}$ " mixing devices support stratification of flow to avoid mixing [25]. 
Table 2. Experimental works on oil-water flow patterns in horizontal pipelines.

\begin{tabular}{|c|c|c|c|c|c|c|c|c|c|c|c|c|c|}
\hline Author & Year & $\begin{array}{c}\text { Diameter } \\
(\mathrm{mm})\end{array}$ & Pipe Material & $\mu_{\mathrm{o}} / \mu_{\mathrm{w}}$ & $\rho_{\mathrm{o}} / \rho_{\mathrm{w}}$ & $\sigma(\mathrm{mN} / \mathrm{m})$ & $\begin{array}{c}\text { Velocity Range } \\
(\mathrm{m} / \mathrm{s})\end{array}$ & $\begin{array}{c}\text { Measurement } \\
\text { Techniques }\end{array}$ & $\begin{array}{c}\text { Observed } \\
\text { Flow Patterns }\end{array}$ & Fluids Used & Temperature/Pressure & Mixing Device & $\mathrm{L} / \mathrm{D}$ \\
\hline Russell et al. [6] & 1959 & 20.5 & $\begin{array}{c}\text { Cellulose } \\
\text { acetate } \\
\text { butyrate }\end{array}$ & 20.13 & 0.834 & 40 & $\begin{array}{l}\mathrm{v}_{\mathrm{so}}=0.01-1.0 \\
\mathrm{v}_{\mathrm{sw}}=0.04-1.08\end{array}$ & $\begin{array}{c}\mathrm{VO}+\mathrm{P}(4 \times 5 \text { in } \\
\text { Linhoof press } \\
\text { camera with a } 90 \\
\text { mm lens })\end{array}$ & $\mathrm{Bb}, \mathrm{ST}$, mixed & $\begin{array}{l}\text { Mineral oil-Kremol } \\
\text { 70/water }\end{array}$ & $25^{\circ} \mathrm{C} / \mathrm{NP}$ & $48^{\circ} \mathrm{Y}$ & 419 \\
\hline Charles et al. [7] & 1961 & 26.4 & $\begin{array}{c}\text { Cellulose } \\
\text { acetate } \\
\text { butyrate }\end{array}$ & $\begin{array}{r}7.04 \\
18.79 \\
72.71 \\
\end{array}$ & 1 & $\begin{array}{l}45, \\
45, \\
30\end{array}$ & $\begin{array}{l}\mathrm{v}_{\mathrm{so}}=0.02-0.9 \\
\mathrm{v}_{\mathrm{sw}}=0.043-1.07 \\
0.043-1.08, \\
0.043-1.09\end{array}$ & $\mathrm{VO}+\mathrm{P}$ & $\begin{array}{l}\text { SL, AN, Bb, } \\
\text { Dw/o, Do/w }\end{array}$ & $\begin{array}{c}\text { Oil (Marcol CX)/water; } \\
\text { Oil (Wyrol J)//water; } \\
\text { Oil (Teresso 85)/water }\end{array}$ & $25^{\circ} \mathrm{C} / \mathrm{NP}$ & $\begin{array}{l}\text { Oil via nozzle, } \\
\text { water via } \\
\text { annulus }\end{array}$ & 277 \\
\hline Hasson et al. [33] & 1970 & 12.6 & Glass-pipe & 1.22 & $\begin{array}{c}\rho_{\mathrm{m}}= \\
1020 \\
\mathrm{~kg} / \mathrm{m}^{3}\end{array}$ & $17-17.5$ & & $\mathrm{VO}+\mathrm{P}$ & $\begin{array}{l}\mathrm{D}, \mathrm{SL}, \mathrm{ST}, \mathrm{AN} \\
\text { and mixed } \\
\text { flows }\end{array}$ & $\begin{array}{l}\text { Kerosene-perchloroethylen } \\
\text { water }\end{array}$ & distilled $30^{\circ} \mathrm{C} / \mathrm{NP}$ & $\begin{array}{c}\text { Inlet nozzle } \\
\text { device for } \\
\text { concentric flow }\end{array}$ & 214 \\
\hline $\begin{array}{l}\text { Guzhov et al. [38] } \\
\text { Source: }[49,50]\end{array}$ & 1973 & 39.4 & Steel & 21.8 & 0.896 & 44.8 & $\mathrm{v}_{\mathrm{m}}=0.3-1.6$ & vo & $\begin{array}{l}\text { ST, ST \& MI, } \\
\text { Do/w, Dw/o, } \\
\text { Do/w \& w/, } \\
\text { Dw/o \& o/w }\end{array}$ & NP & $21^{\circ} \mathrm{C} / \mathrm{NP}$ & NP & $\mathrm{NP}$ \\
\hline Oglesby [20] & 1979 & 41 & Steel & $\begin{array}{l}167, \\
61, \\
32\end{array}$ & $\begin{array}{l}0.87 ; \\
0.863 ; \\
0.859\end{array}$ & 35.4 & $\mathrm{NP}$ & vo & $\begin{array}{c}\text { ST, ST \& MI, } \\
\text { Do/w \& w, o/w, } \\
\text { Dw/o \& Do/w, } \\
\text { w/o, SL, SLw, } \\
\text { AN, AO }\end{array}$ & $\begin{array}{c}\text { Oil (Sun Oil Co. solvent } \\
\text { (250-SN)/water; } \\
\text { Oil (diesel fuel)/water; } \\
\text { Oil (blend of Sun Oil Co. } \\
\text { solvent }(250-\mathrm{SN} \text { and } \\
\text { diesel fuel)/water }\end{array}$ & $\begin{array}{c}\mathrm{NP} ; 18.3^{\circ} \mathrm{C} / \mathrm{NP} ; 21.1 \\
{ }^{\circ} \mathrm{C} / \mathrm{NP} .\end{array}$ & $90^{\circ}$ angle 2 in $\mathrm{T}$ & 282 \\
\hline Arirachakaran et al. [17] & 1989 & 41 & Steel & $\begin{array}{l}27.9, \\
344, \\
498, \\
682\end{array}$ & $\begin{array}{l}0.854, \\
0.868, \\
0.868, \\
0.868\end{array}$ & $\begin{array}{l}\mathrm{NP}, \\
29, \\
30, \\
31\end{array}$ & $\mathrm{v}_{\mathrm{m}}=0.457-3.66$ & NP & $\begin{array}{l}\text { ST, Do/w \& w, } \\
\text { o/w, o \& Dw/o, } \\
\text { w/o, SL, SLw, } \\
\text { AN, AO }\end{array}$ & $\begin{array}{l}\text { No. } 2 \text { Diesel fuel oil/tap } \\
\text { water, } \\
\text { Experimental oil No. } \\
\text { 1/tap water, } \\
\text { Experimental oil No. } \\
\text { 2/tap water, } \\
\text { Experimental oil No. } \\
\text { 3/tap water } \\
\end{array}$ & $21.1^{\circ} \mathrm{C} / \mathrm{NP}$ & NP & 312 \\
\hline Arirachakaran et al. [17] & 1989 & 26.6 & Steel & $\begin{array}{r}1405 \\
12550\end{array}$ & $\begin{array}{l}0.869, \\
0.90\end{array}$ & $\begin{array}{l}32, \\
32\end{array}$ & $\mathrm{v}_{\mathrm{m}}=1.5-3.05$ & NP & $\begin{array}{l}\text { ST, Do/w \& w, } \\
\text { o/w, o \& Dw/o, } \\
\text { w/o, SL, SLw, } \\
\text { AN, AO }\end{array}$ & $\begin{array}{c}\text { Refined oil (SN-250)/tap } \\
\text { water } \\
\text { Refined oil (150-SB)/tap } \\
\text { water }\end{array}$ & $21.1^{\circ} \mathrm{C} / \mathrm{NP}$ & NP & 229 \\
\hline Kurban et al. [51] & 1995 & $24.3 ; 24$ & Glass, Acrylic & 1.6 & 0.803 & $\mathrm{NP}$ & NP & $\begin{array}{c}\mathrm{P}+\text { conductivity } \\
\text { probe }\end{array}$ & $\begin{array}{c}\text { ST, ST \& MI, } \\
\text { Dw/o }\end{array}$ & NP & NP & $\mathrm{NP}$ & $\mathrm{NP}$ \\
\hline Trallero et al. [11] & 1997 & 50.1 & Acrylic resin & 29.7 & 0.852 & 36 & $\begin{array}{l}\mathrm{v}_{\mathrm{so}}=0.01-1.61 \\
\mathrm{v}_{\mathrm{sw}}=0.01-1.83\end{array}$ & vo & $\begin{array}{c}\text { ST, ST \& MI, } \\
\text { Do/w \& w, o/w, } \\
\text { Dw/o \& Do/w, } \\
\text { o/w }\end{array}$ & $\begin{array}{l}\text { Mineral oil (Crystex } \\
\text { AF-M)/tap water }\end{array}$ & $25.5^{\circ} \mathrm{C} / \mathrm{NP}$ & Y-type & 310 \\
\hline Nädler and Mewes [21] & 1997 & 59 & Perspex & $22-35$ & 0.848 & NP & $\mathrm{v}_{\mathrm{m}}=0.3-1.61$ & $\begin{array}{c}\mathrm{VO}+\text { conductance } \\
\text { probe }\end{array}$ & $\begin{array}{c}\text { ST, ST \& MI, } \\
\text { Do/w \& w, o/w, } \\
\text { Dw/o \& Do/w } \\
\& \text { w, Dw/o \& } \\
\text { w, w/o. }\end{array}$ & $\begin{array}{l}\text { Mineral white oil (Shell } \\
\text { Ondina 17)/tap water }\end{array}$ & $30^{\circ} \mathrm{C}, 25^{\circ} \mathrm{C}, 18^{\circ} \mathrm{C} / \mathrm{NP}$ & $\begin{array}{l}\text { Cone-shaped } \\
\text { nozzle separated } \\
\text { by baffle plates }\end{array}$ & $\begin{array}{l}225, \\
680\end{array}$ \\
\hline
\end{tabular}


Table 2. Cont.

\begin{tabular}{|c|c|c|c|c|c|c|c|c|c|c|c|c|c|}
\hline Author & Year & $\begin{array}{c}\text { Diameter } \\
(\mathrm{mm})\end{array}$ & Pipe Material & $\mu_{0} / \mu_{w}$ & $\rho_{0} / \rho_{w}$ & $\sigma(\mathrm{mN} / \mathrm{m})$ & $\begin{array}{c}\text { Velocity Range } \\
(\mathrm{m} / \mathrm{s})\end{array}$ & $\begin{array}{l}\text { Measurement } \\
\text { Techniques }\end{array}$ & $\begin{array}{c}\text { Observed } \\
\text { Flow Patterns }\end{array}$ & Fluids Used & Temperature/Pressure & Mixing Device & L/D \\
\hline Vedapuri, et al. [36] & 1997 & 101.6 & Plexiglass & $2 ; 90$ & & NP & $\mathrm{v}_{\mathrm{m}}=0.1-2.0$ & $\begin{array}{l}\text { VO (by video home } \\
\text { system camera) }\end{array}$ & $\begin{array}{l}\text { ST \& MI, Dw/o } \\
\& \& \mathrm{o} / \mathrm{w}\end{array}$ & $\begin{array}{l}\text { Oil (Standard ASTM } \\
\text { salt-water)/water }\end{array}$ & $40^{\circ} \mathrm{C} / 0.13 \mathrm{MPa}$ & T-junction & 177 \\
\hline Valle and Utvik [35] & 1997 & 77.9 & Steel & 1 & 0.741 & NP & $\mathrm{v}_{\mathrm{m}}=1.17,1.74,2.33$ & $\begin{array}{l}\text { Conductivity needle } \\
\text { probe }\end{array}$ & Do/w, Dw/o, S & $\begin{array}{l}\text { Light crude oil/synthetic } \\
\text { formation water }\end{array}$ & $70^{\circ} \mathrm{C} / 10.5 \mathrm{MPa}$ & $\mathrm{NP}$ & 154 \\
\hline Bannwart [43] & 1998 & 22.5 & Glass & 2700 & 0.989 & 40 & $\begin{array}{l}\mathrm{v}_{\mathrm{so}}=0.30-0.63, \mathrm{v}_{\mathrm{sw}} \\
=0.03-0.28\end{array}$ & $\begin{array}{l}\text { P(Kodak EktaPro } \\
\text { EM high speed } \\
\text { camera) }\end{array}$ & AN & Viscous oil/water & $\mathrm{RT} / \mathrm{NP}$ & $\begin{array}{l}\text { Nozzle (tube for } \\
\text { oil, shell for } \\
\text { water) }\end{array}$ & NP \\
\hline Soleimani [52] & 1999 & 25.4 & Steel & 1.6 & 0.801 & $17 @ 22{ }^{\circ} \mathrm{C}$ & $\mathrm{v}_{\mathrm{m}}=1.25-3.0$ & $\begin{array}{l}\text { VO, Impedance } \\
\text { probe, Gamma } \\
\text { densitometer }\end{array}$ & $\begin{array}{l}\text { ST, SWD, } \\
\text { SMW, SMO, } \\
\text { 3L, Dw/o \& } \\
\text { Do/w }\end{array}$ & $\begin{array}{l}\text { Kerosene/(Exxsol } \\
\text { D80)/tap water }\end{array}$ & $25^{\circ} \mathrm{C} / \mathrm{NP}$ & Static mixer & 382 \\
\hline Angeli and Hewitt [30] & 2000 & $24.3 ; 24$ & $\begin{array}{l}\text { Steel; Acrylic } \\
\text { resin }\end{array}$ & 1.6 & 0.801 & 17 & $\mathrm{v}_{\mathrm{m}}=0.2-3.9$ & $\begin{array}{l}\text { VO, high-speed } \\
\text { video-camera, HFI } \\
\text { probe }\end{array}$ & $\begin{array}{c}\text { SW, SWD, } \\
\text { SMO, 3L, } \\
\text { SMW, Mixed }\end{array}$ & $\begin{array}{l}\text { Kerosene (Exxsol D80)/tap } \\
\text { water }\end{array}$ & $20^{\circ} \mathrm{C} / \mathrm{NP}$ & T-junction & $\begin{array}{l}370 ; \\
375\end{array}$ \\
\hline $\begin{array}{l}\text { Simmons and Azzopardi } \\
{[37]}\end{array}$ & 2001 & 63 & PVC & 1.125 & 0.684 & 10 & $\mathrm{v}_{\mathrm{m}}=0.8-3.1$ & $\begin{array}{l}\text { Lasentec TM Par-Tec } \\
300 \text { C, Malvern } 2600\end{array}$ & $\begin{array}{l}\mathrm{SM}, \mathrm{Dw} / \mathrm{o} \& \mathrm{w}, \\
\text { Dw/o }\end{array}$ & $\begin{array}{c}\text { Kerosene/Aqueous } \\
\text { potassium carbonate } \\
\text { solution } \\
\end{array}$ & NP & $\begin{array}{l}\text { Mixer with } \\
\text { porous walls }\end{array}$ & 71 \\
\hline $\begin{array}{c}\text { Mu [53] } \\
\text { Source: [54] }\end{array}$ & 2001 & 25.4 & Steel & 310 & 0.93 & $\mathrm{NP}$ & NP & High-speed P & $\begin{array}{c}\text { ST, ST \& MI, } \\
\text { SLw, AO Do/w, } \\
\text { Dw/o }\end{array}$ & $\mathrm{NP}$ & $\mathrm{NP}$ & $\mathrm{NP}$ & NP \\
\hline Bannwart et al. [42] & 2004 & 28.4 & Glass & 488.0 & 0.926 & 29 & $\begin{array}{l}\mathrm{v}_{\mathrm{so}}=0.007-2.5 ; \mathrm{vsw} \\
=0.04-0.5\end{array}$ & $\begin{array}{c}\mathrm{P}(\text { high speed } \\
\text { camera (Olympus, } \\
1000 \text { frames/s) (at } \\
\text { slow motion } 30 \\
\text { frames/s) }\end{array}$ & $\mathrm{ST}, \mathrm{Bb}, \mathrm{AN}$ & Heavy crude oil/water & $20^{\circ} \mathrm{C} / \mathrm{NP}$ & $\begin{array}{l}\text { Injection nozzle } \\
\text { (central tube for } \\
\text { oil, shell for } \\
\text { water) } \\
\end{array}$ & 191 \\
\hline Lovick and Angeli [55] & 2004 & 38 & Stainless steel & 6.0 & 0.828 & 39.6 & $\mathrm{v}_{\mathrm{m}}=0.8-3.0$ & $\begin{array}{l}\text { VO, conductance } \\
\text { probe and HFI } \\
\text { probe }\end{array}$ & $\begin{array}{l}\text { SW, Dw/o, } \\
\text { Do/w, DC }\end{array}$ & $\begin{array}{c}\text { Mineral oil } \\
\text { (ExxsolD140)/tap water }\end{array}$ & NP & $\begin{array}{l}\mathrm{T} \text { (mixing } \\
\text { reduction) }\end{array}$ & 210 \\
\hline Chakrabarti et al. [56] & 2005 & 25.4 & $\begin{array}{l}\text { Polymethyl } \\
\text {-methacrylate }\end{array}$ & 0.7 & 0.787 & 45 & $\begin{array}{l}\mathrm{V}_{\mathrm{so}}=0.029-2.12 \\
\mathrm{v}_{\mathrm{sw}}=0.04-1.46\end{array}$ & $\mathrm{VO}+\mathrm{P}$ & $\begin{array}{l}\text { ST, ST \& MI, } \\
\text { PL, Do/w \& w, } \\
\text { Dw/o \& o }\end{array}$ & Kerosene/water & $25^{\circ} \mathrm{C} / \mathrm{NP}$ & $\begin{array}{l}\text { Mixer (pipe } \\
\text { inserted in } \\
\text { another pipe) }\end{array}$ & 84 \\
\hline $\begin{array}{l}\text { Wegmann and Rudolf } \\
\text { von Rohr [34] }\end{array}$ & 2006 & $5.6 ; 7$ & Glass & $5.78-6.14$ & $0.82-0.822$ & $62.2 @ 20^{\circ} \mathrm{C}$ & $\begin{array}{l}\mathrm{v}_{\mathrm{so}}=0.01-2.5, \\
\mathrm{v}_{\mathrm{sw}}=0.01-2.0 \\
\mathrm{v}_{\mathrm{v}_{\mathrm{s}}}=0.01-1.6, \\
\mathrm{v}_{\mathrm{sw}}=0.01-13 .\end{array}$ & $\begin{array}{l}\text { P (digital camera } \\
\text { (Minolta Dimage } 7 \mathrm{i} \\
\text { with a resolution of } \\
2560-1920 \text { pixels) }\end{array}$ & $\begin{array}{l}\text { ST, AN, I, } \\
\text { Do/w, Dw/o }\end{array}$ & Paraffin-oil/water & $19.1-23.3^{\circ} \mathrm{C} / \mathrm{NP}$ & $\begin{array}{l}\text { T-shaped } \\
\text { (selected to } \\
\text { prevent } \\
\text { emulsion } \\
\text { formation }\end{array}$ & $\begin{array}{l}660 ; \\
528\end{array}$ \\
\hline Vielma et al. [57] & 2007 & 50.8 & Acrylic resin & 18.8 & 0.859 & 16.4 & $\begin{array}{l}\mathrm{v}_{\mathrm{so}}=0.03-1.75, \mathrm{v}_{\mathrm{sw}} \\
=0.03-1.75\end{array}$ & $\begin{array}{l}\mathrm{VO}, \mathrm{P} \text { and } \\
\text { conductance probe }\end{array}$ & $\begin{array}{c}\text { ST, ST \& MI, } \\
\text { Do/w \& w, o/w, } \\
\text { Dw/o \& Do/w, } \\
\text { o/w }\end{array}$ & $\begin{array}{l}\text { Refined mineral oil (Tulco } \\
\text { Tech 80)/tap water }\end{array}$ & $40^{\circ} \mathrm{C}-29^{\circ} \mathrm{C} / \mathrm{NP}$ & $30^{\circ} \mathrm{Y}$ & 440 \\
\hline
\end{tabular}


Table 2. Cont.

\begin{tabular}{|c|c|c|c|c|c|c|c|c|c|c|c|c|c|}
\hline Author & Year & $\begin{array}{c}\text { Diameter } \\
(\mathrm{mm})\end{array}$ & Pipe Material & $\mu_{\mathrm{o}} / \mu_{\mathrm{w}}$ & $\rho_{\mathrm{o}} / \rho_{\mathrm{w}}$ & $\sigma(\mathrm{mN} / \mathrm{m})$ & $\begin{array}{c}\text { Velocity Range } \\
(\mathrm{m} / \mathrm{s})\end{array}$ & $\begin{array}{l}\text { Measurement } \\
\text { Techniques }\end{array}$ & $\begin{array}{c}\text { Observed } \\
\text { Flow Patterns }\end{array}$ & Fluids Used & Temperature/Pressure & Mixing Device & L/D \\
\hline Grassi et al. [41] & 2008 & 21 & Polycarbonate & 615 & 0.886 & 50 & $\begin{array}{l}\mathrm{v}_{\mathrm{so}}=0.029-0.7 \\
\mathrm{v}_{\mathrm{sw}}=0.14-2.5\end{array}$ & $\mathrm{VO}+\mathrm{P}$ & $\begin{array}{l}\text { ST, Do/w, AN, } \\
\text { PL/SL, Do/w \& } \\
\text { w }\end{array}$ & Paraffin oil/water & $20^{\circ} \mathrm{C} / \mathrm{NP}$ & $\begin{array}{l}\text { Injecting device } \\
\text { (oil at the core } \\
\text { and water } \\
\text { annulus) }\end{array}$ & 429 \\
\hline Dasari et al. [58] & 2013 & 25 & Perspex & 107 & 0.889 & 24 & $\begin{array}{l}\mathrm{v}_{\mathrm{so}}=0.015-1.22, \\
\mathrm{v}_{\mathrm{sw}}=0.1-1.1\end{array}$ & $\begin{array}{c}\mathrm{VO}+\mathrm{P} \text { (camera } \\
\text { model no } \\
\text { DSC-HX100 V, by } \\
\text { sony) }\end{array}$ & $\begin{array}{l}\text { SL, ST, ST \& } \\
\text { MI, Do/w, } \\
\text { Dw/o, PL }\end{array}$ & Lube oil/water & $25^{\circ} \mathrm{C} / \mathrm{NP}$ & NP & 40 \\
\hline Al-Wahaibi et al. [22] & 2014 & 19 & Acrylic & 12 & 0.877 & 20.1 & $\begin{array}{l}\mathrm{v}_{\mathrm{so}}=0.06-1.69, \mathrm{v}_{\mathrm{sw}} \\
=0.12-1.69\end{array}$ & $\begin{array}{c}\mathrm{VO}+\text { High speed } \\
\text { camera (Fastec } \\
\text { Troubleshooter, } \\
\text { records up to } 1000 \\
\text { fps) } \\
\end{array}$ & $\begin{array}{l}\mathrm{ST}, \mathrm{Bb}, \mathrm{AN}, \\
\mathrm{DC}, \mathrm{Do} / \mathrm{w}, \\
\mathrm{DW} w / \mathrm{o}\end{array}$ & Mineral oil/water & $\mathrm{NP}$ & Y-junction & 421 \\
\hline Voulgaropoulos et al. [59] & 2016 & 37 & Acrylic & 6.2 & 0.830 & 32.9 & $\mathrm{v}_{\mathrm{m}}=0.31-0.73$ & $\begin{array}{l}\text { High-speed camera } \\
\text { (with Tokina Macro } \\
\text { lens) and a dual } \\
\text { conductance probe }\end{array}$ & $\begin{array}{l}\text { ST, SM, Do/w, } \\
\text { Dw/o, Do/w \& } \\
\text { w, Dw/o \& o/w }\end{array}$ & $\begin{array}{l}\text { Kerosene (Exxsol } \\
\text { D140)/fresh water }\end{array}$ & $20^{\circ} \mathrm{C} / \mathrm{NP}$ & $\begin{array}{l}\text { Multi-nozzle } \\
\text { inlet }\end{array}$ & 189 \\
\hline
\end{tabular}

Note: $\mathrm{v}_{\mathrm{so}}=$ Superficial oil velocity, $\mathrm{v}_{\mathrm{sw}}=$ Superficial water velocity, $\mathrm{v}_{\mathrm{m}}=$ Mixture velocity, $\mathrm{VO}=$ Visual Observation, $\mathrm{P}=\mathrm{Photography}, \mathrm{HFI}=\mathrm{High}$-frequency impedance, NP $=$ Not

provided $\mathrm{L} / \mathrm{D}=$ the ratio of part of the test section in which testing actual took place or the length of the test section from entrance to inner diameter of the pipe, RT $=$ room temperature, $\rho_{\mathrm{m}}$ $=$ mixture density, $\mu_{\mathrm{o}} / \mu_{\mathrm{w}}=$ ratio of oil viscosity to water viscosity, $\rho_{\mathrm{o}} / \rho_{\mathrm{W}}=$ ratio of oil density to water density. Note: $3 \mathrm{~L}:$ Three layers; AN: Annular/oil in water concentric; AO: Annular/oil annulus; Bb: Oil bubbles in water; D: Dispersed flow; DC: Dual continuous; Do/w: Dispersed oil in water; Dw/o: Dispersed water in oil; Do/w \& w: Dispersion of oil in water with water layer; Dw/o \& Do/w: Dispersed water-in-oil and oil-in-water; Dw/o \& Do/w \& w: Dispersed water in oil and dispersed oil in water, and water layer; I: Intermediate flow; M: Mixed; o/w: Oil-in-water emulsion; O \& W/O: Oil layer and dispersed water in oil; PL: Plug flow; SL: Oil slugs in water; SLw: Water slugs in oil; SMO: Stratified mixed/oil; ST: Stratified flow; ST \& MI: Stratified flow with mixing at the interface; SW: Stratified wavy; SWD: Stratified wavy with droplets at the interface (Stratified wavy/drops); SMW: Stratified mixed/water; w/o: Water-in-oil emulsion. 
A dimensionless length $(\mathrm{L} / \mathrm{D})$ is defined as the ratio of the length of the pipeline from the entrance to the observation point (sometimes equal to the length of the test section) to the pipe diameter. The $\mathrm{L} / \mathrm{D}$ is a critical parameter for guaranteeing fully developed flow conditions [25].

Mukhaimer et al. [18] showed the effect of water salinity on flow patterns in an oil-water horizontal pipeline. They achieved water salinity of $75 \%$ o by adding common salt ( $9 \%$ water weight) to the water tank. Due to this addition of salt, water density changed from $999 \mathrm{~kg} / \mathrm{m}^{3}$ to $1065 \mathrm{~kg} / \mathrm{m}^{3}$, while viscosity changed from $0.985 \mathrm{mPa}$ s to $1.246 \mathrm{mPa}$ s at $23.5^{\circ} \mathrm{C}$. Within a velocity range of $0.05-3 \mathrm{~m} / \mathrm{s}$ for oil-tap water flow, seven flow patterns were observed, including: dispersed oil in water (Do/w), dispersed oil in water with water layer (Do/w \& w), dispersed water in oil (Dw/o), dispersed water in oil and dispersed oil in water (Dw/o \& o/w), stratified (ST), stratified with mixing at interface (ST \& MI), and dispersed water in oil with oil layer (Dw/o \& o). For the same velocity range, Dw/o \& o was not observed with oil-saline water flow. They also observed that salinity delayed the transition from $\mathrm{Do} / \mathrm{w}$ \& $w$ to Dw/o \& o.

\subsection{Development of Flow Maps for Liquid-Liquid in a Horizontal Pipeline}

Various researchers have constructed flow maps for liquid-liquid flow in horizontal pipelines in several ways. Table 3 presents the chronological development of the liquid-liquid flow map.

Table 3. Development of flow maps for liquid-liquid in a horizontal pipeline.

\begin{tabular}{|c|c|c|c|}
\hline Author & Year & Mapping Parameters & Identified Flow Pattern \\
\hline Russell et al. [6] & 1959 & Friction factor $\left(f_{\mathrm{f}}\right)$ versus superficial water velocity $\left(\mathrm{v}_{\mathrm{SW}}\right)$ & $\mathrm{Bb}, \mathrm{ST}$, mixed \\
\hline Charles et al. [7] & 1961 & $\begin{array}{l}\text { Superficial oil velocity }\left(\mathrm{v}_{\mathrm{so}}\right) \text { versus superficial water } \\
\text { velocity }\left(\mathrm{v}_{\mathrm{sw}}\right)\end{array}$ & $\mathrm{w} / \mathrm{o}, \mathrm{AN}, \mathrm{SL}, \mathrm{Bb}$, and o/w \\
\hline Hasson et al. [33] & 1970 & $\begin{array}{l}\text { The organic phase flow rate }\left(\mathrm{Q}_{\mathrm{or}}\right) \text { versus the water phase } \\
\text { flow rate }\left(\mathrm{Q}_{\mathrm{w}}\right)\end{array}$ & $\mathrm{D}, \mathrm{SL}, \mathrm{ST}, \mathrm{AN}$ and mixed flows \\
\hline $\begin{array}{l}\text { Guzhov et al. [38] } \\
\text { Source: }[49,50]\end{array}$ & 1973 & Mixture velocity $\left(\mathrm{v}_{\mathrm{m}}\right)$ versus input water fraction $\left(\mathrm{f}_{\mathrm{w}}\right)$ & $\begin{array}{l}\text { ST, ST \& MI, Do/w, Dw/o, Do/w \& } \\
\text { w, Dw/o \& o/w }\end{array}$ \\
\hline Oglesby [20] & 1979 & Mixture velocity $\left(\mathrm{v}_{\mathrm{m}}\right)$ versus input water fraction $\left(\mathrm{f}_{\mathrm{w}}\right)$ & $\begin{array}{l}\text { ST, ST \& MI, Do/w \& w, o/w, Dw/o } \\
\text { \& Do/w, w/o, SL, SLw, AN, AO }\end{array}$ \\
\hline Arirachakaran et al. [17] & 1989 & Mixture velocity $\left(\mathrm{v}_{\mathrm{m}}\right)$ versus input water fraction $\left(\mathrm{f}_{\mathrm{w}}\right)$ & $\begin{array}{l}\text { ST, Do/w \& w, o/w, o \& Dw/o, w/o, } \\
\text { SL, SLw, AN, AO }\end{array}$ \\
\hline Vedapuri et al. [36] & 1997 & $\begin{array}{l}\text { The thickness of the water layer to the diameter of the pipe, } \\
\text { h/D against the percentage of water (input water fraction } f_{w} \text { ) }\end{array}$ & $\begin{array}{l}\text { Semi-segregated, semi-mixed and } \\
\text { semi-dispersed }\end{array}$ \\
\hline Trallero et al. [11] & 1997 & $\begin{array}{l}\text { 1. Superficial water velocity }\left(\mathrm{v}_{\mathrm{sw}}\right) \text { versus superficial oil } \\
\text { velocity }\left(\mathrm{v}_{\mathrm{so}}\right) \\
\text { 2. Mixture velocity }\left(\mathrm{v}_{\mathrm{m}}\right) \text { versus input water fraction }\left(\mathrm{f}_{\mathrm{w}}\right)\end{array}$ & $\begin{array}{l}\text { ST, ST \& MI, Do/w \& w, o/w, Dw/o } \\
\qquad \mathrm{Do} / \mathrm{w}, \mathrm{o} / \mathrm{w}\end{array}$ \\
\hline Nädler and Mewes [21] & 1997 & $\begin{array}{l}\text { 1. Superficial oil velocity }\left(\mathrm{v}_{\mathrm{so}}\right) \text { versus superficial water } \\
\text { velocity }\left(\mathrm{v}_{\mathrm{sw}}\right) \\
\text { 2. Mixture velocity }\left(\mathrm{v}_{\mathrm{m}}\right) \text { versus input water fraction }\left(\mathrm{f}_{\mathrm{W}}\right)\end{array}$ & $\begin{array}{l}\text { ST, ST \& MI, Do/w \& w, o/w, Dw/o } \\
\quad \& \text { Do/w \& w, Dw/o \& w, w/o }\end{array}$ \\
\hline Angeli and Hewitt [30] & 2000 & $\begin{array}{l}\text { Mixture velocity }\left(\mathrm{v}_{\mathrm{m}}\right) \text { against input water volume } \\
\text { fraction }\left(\mathrm{f}_{\mathrm{w}}\right)\end{array}$ & SW, SWD, SMO, 3L, SMW, Mixed \\
\hline Brauner [2] & 2003 & (No map proposed) Eotvös number & $\begin{array}{l}\text { Gravity force dominated or } \\
\text { interfacial force dominated }\end{array}$ \\
\hline Lovick and Angeli [55] & 2004 & Mixture velocity $\left(\mathrm{v}_{\mathrm{m}}\right)$ against input oil volume fraction $\left(\mathrm{f}_{\mathrm{o}}\right)$ & Dw/o, Do/w, DC, SW \\
\hline Chakrabarti et al. [56] & 2005 & $\begin{array}{l}\text { Superficial water velocity }\left(\mathrm{v}_{\mathrm{sw}}\right) \text { versus superficial oil } \\
\text { velocity }\left(\mathrm{v}_{\mathrm{so}}\right)\end{array}$ & $\begin{array}{c}\text { ST, SW, 3L, Do/w \& w, D, PL, O \& } \\
\text { W/O }\end{array}$ \\
\hline $\begin{array}{l}\text { Wegmann and Rudolf } \\
\text { von Rohr [34] }\end{array}$ & 2006 & $\begin{array}{l}\text { Mixture velocity }\left(\mathrm{v}_{\mathrm{m}}\right) \text { against input water volume } \\
\text { fraction }\left(\mathrm{f}_{\mathrm{w}}\right)\end{array}$ & ST, AN, I, Do/w, Dw/o \\
\hline Vielma et al. [57] & 2007 & $\begin{array}{l}\text { Superficial water velocity }\left(\mathrm{v}_{\mathrm{sw}}\right) \text { versus superficial oil } \\
\text { velocity }\left(\mathrm{v}_{\mathrm{so}}\right)\end{array}$ & $\begin{array}{l}\text { ST, ST \& MI, Do/w \& w, o/w, Dw/o } \\
\text { \& Do/w, o/w }\end{array}$ \\
\hline Grassi et al. [41] & & $\begin{array}{l}\text { Superficial water velocity }\left(\mathrm{v}_{\mathrm{sw}}\right) \text { versus superficial oil } \\
\text { velocity }\left(\mathrm{v}_{\mathrm{so}}\right)\end{array}$ & ST, Do/w, AN, PL/SL, Do/w \& w \\
\hline Hapanowic [60] & 2010 & $\begin{array}{l}\text { Superficial mass fluxes for oil }\left(\mathrm{g}_{\mathrm{ol}, \mathrm{o}}\right)\left[\mathrm{kg} / \mathrm{m}^{2} . \mathrm{s}\right] \text { versus } \\
\text { Superficial mass fluxes for water }\left(\mathrm{g}_{\mathrm{w}, \mathrm{o}}\right)\left[\mathrm{kg} / \mathrm{m}^{2} . \mathrm{s}\right]\end{array}$ & $\begin{array}{l}\text { Dr-drops, DrP-drops and plugs, } \\
\text { D-dispersion, S-stratification } \\
\text { AD-annular and dispersion; } \\
\text {-W-for water, -O-for oil }\end{array}$ \\
\hline
\end{tabular}


Table 3. Cont.

\begin{tabular}{|c|c|c|c|}
\hline Author & Year & Mapping Parameters & Identified Flow Pattern \\
\hline Yusuf et al. [29] & 2012 & $\begin{array}{l}\text { Superficial water velocity }\left(\mathrm{v}_{\mathrm{sw}}\right) \text { versus superficial oil } \\
\text { velocity }\left(\mathrm{v}_{\mathrm{so}}\right)\end{array}$ & $\mathrm{ST}, \mathrm{Bb}, \mathrm{AN}, \mathrm{DC}, \mathrm{Do} / \mathrm{w}, \mathrm{Dw} / \mathrm{o}$ \\
\hline Dasari et al. [58] & 2013 & $\begin{array}{l}\text { Superficial water velocity }\left(\mathrm{v}_{\mathrm{sw}}\right) \text { versus superficial oil } \\
\text { velocity }\left(\mathrm{v}_{\mathrm{so}}\right)\end{array}$ & SL, ST, ST \& MI, Do/w, Dw/o, PL \\
\hline Al-Wahaibi et al. [22] & 2014 & $\begin{array}{l}\text { Superficial water velocity }\left(\mathrm{v}_{\mathrm{sw}}\right) \text { versus superficial oil } \\
\text { velocity } \mathrm{v}_{\mathrm{so}}\end{array}$ & $\mathrm{ST}, \mathrm{Bb}, \mathrm{AN}, \mathrm{DC}, \mathrm{Do} / \mathrm{w}, \mathrm{Dw} / \mathrm{o}$ \\
\hline Ibarra et al. [5] & 2015 & $\left(\operatorname{Re}_{\mathrm{m}} / \mathrm{Eo}\right) \operatorname{versus}\left(\mathrm{f}_{\mathrm{w}}\right)$ & ST, SWD, Do/w \& w, DC, D \\
\hline Shi and Yeung [61] & 2017 & $\begin{array}{l}\text { The gravitation to viscous force ratio }(G / V) \text { versus input } \\
\text { water volume fraction }\left(f_{W}\right)\end{array}$ & ST, D, I, CAF \\
\hline \multicolumn{4}{|c|}{$\begin{array}{l}\text { Note: 3L: Three layers; AN: Oil in water concentric; AO: Annular/oil annulus; Bb: Oil bubbles in water; D: Dispersed } \\
\text { flow; DC: Dual continuous; Do/w: Dispersed oil in water; Dw/o: Dispersed water in oil; Do/w \& w: Dispersion of } \\
\text { oil in water with water layer; Dw/o \& Do/w: Dispersed water-in-oil and oil-in-water; Dw/o \& Do/w \& w: Dispersed } \\
\text { water in oil and dispersed oil in water, and water layer; I: Intermediate flow; M: Mixed; o/w: Oil-in-water emulsion; } \\
\text { O \& W/O: Oil layer and dispersed water in oil; PL: Plug flow; SL: Oil slugs in water; SLw: Water slugs in oil; SMO: } \\
\text { Stratified mixed/oil; ST: Stratified flow; ST \& MI: Stratified flow with mixing at the interface; SW: Stratified wavy; } \\
\text { SWD: Stratified wavy with droplets at the interface (Stratified wavy/drops); SMW: Stratified mixed/water; w/o: } \\
\text { Water-in-oil emulsion. }\end{array}$} \\
\hline
\end{tabular}

Russell et al. [6] were one of the first groups to characterise a liquid-liquid flow using white mineral oil, with a density of $835 \mathrm{~kg} / \mathrm{m}^{3}$ and a viscosity of $18 \mathrm{mPa}$ s, and water in an $8 \mathrm{~m}$ long horizontal, smooth, 1-inch pipe. They investigated the flow conditions over a range of input oil-water volume ratios ranging from 0.1 to 10 , and at 13 different superficial water velocities ranging from $0.04 \mathrm{~m} / \mathrm{s}$. to $1.08 \mathrm{~m} / \mathrm{s}$. Three flow patterns were identified (bubble, stratified and mixed flows) by plotting the frictional factor $\left(f_{f}\right)$ versus the superficial water velocity $\left(v_{s w}\right)$. They showed that these flow patterns occurred in laminar, transitional or turbulent conditions of flow.

Charles et al. [7] and Nädler and Mewes [21] plotted superficial oil velocity $\left(\mathrm{v}_{\mathrm{so}}\right)$ versus superficial water velocity $\left(\mathrm{v}_{\mathrm{sw}}\right)$ to express the flow configurations observed in their experiments. Charles et al. [7] performed their studies using water and three oils of equal density $\left(\rho_{\mathrm{o}}=998 \mathrm{~kg} / \mathrm{m}^{3}\right)$, but with viscosities of $6.29,16.8$, and $65 \mathrm{mPa}$ s, in a horizontal pipe with $8.78 \mathrm{~m}$ test section and 1-inch internal diameter. These researchers did not observe a stratified flow as there was no difference between the oil and water densities used, which is ordinarily responsible for gravity forces to separate the heavier phase from the lighter phase. With the three different oils, the researchers observed a similar series of flow patterns and concluded that oil viscosity has little effects on flow patterns, although the oil with $65 \mathrm{mPa}$ s viscosity showed different behaviour at high oil-water input ratios. Nädler and Mewes [21] used tap water and mineral white oil of viscosity $20 \mathrm{mPa}$ s and density $841 \mathrm{~kg} / \mathrm{m}^{3}$ in a perspex horizontal pipe with $59 \mathrm{~mm}$ internal diameter. The total length of the pipeline between the entrance nozzle and the separation unit was approximately $48 \mathrm{~m}$. The liquids were introduced into the set-up via a cone-shaped nozzle which had three sections separated by baffle plates to prevent mixing of phases and emulsion formation at the inlet.

Hasson et al. [33] obtained flow pattern maps by plotting the organic phase flow rate ( $\left.Q_{\text {or }}\right)$ versus the water phase flow rate $\left(Q_{w}\right)$. They focused on concentric flow study using distilled water and kerosene-perchloroethylene (PCE) solution, which was mixed to give a density of $1020 \mathrm{~kg} / \mathrm{m}^{3}$, and the viscosity of 0.82 and $1 \mathrm{mPa}$ s for water and the organic, respectively. The two liquids were introduced to a $2.7 \mathrm{~m}$ long horizontal pipe with $12.6 \mathrm{~mm}$ inner diameter via an inlet nozzle device providing initial concentric flow. With the water phase formed at the core being the lighter phase, the heavier organic phase formed the annulus. They concluded that annular flow breakup could occur by two mechanisms; the collapse of the core-liquid by varicose Raleigh type waves or rupture of the top-wall annulus-liquid, diminished by the rise of the lighter core-liquid.

Mixture velocity $\left(\mathrm{v}_{\mathrm{m}}\right)$ versus input water fraction $\left(f_{\mathrm{w}}\right)$ has been used extensively by many researchers to express the flow configuration observed in a liquid-liquid flow in horizontal pipeline $[11,17,20,21,30,34,38,55]$. Guzhov et al. [38] did not observe the annular flow pattern, probably due to a low oil viscosity used for the experiment. Oglesby [20] identified 14 flow patterns using water and three oils with viscosities of 32, 61 and $167 \mathrm{mPa}$ s in a $41 \mathrm{~mm}$ ID horizontal pipe. Annular flow 
with the water phase as core and oil phase as annulus was observed by Arirachakaran et al. [17] and Oglesby [20], which negates the observation of Joseph et al. [62] that the low-viscous liquid always encapsulates the high-viscous liquid. Joseph et al. [62] explained this observation for minimal tractions and dissipation of the low-viscous liquid should be in the zone of high shear. Angeli and Hewitt [30], Guzhov et al. [38], Lovick and Angeli [55], Nädler and Mewes [21], and Trallero et al. [11] did not observe the annular flow, probably due to low viscosity of the oils used in their experiments, which was less than $35 \mathrm{mPa}$ s [15,54], so their oils were neither viscous nor dense enough to create a stable oil core and water annulus [63]. Trallero et al. [11] investigated both experimentally and theoretically by using mineral oil and water as working fluids whose properties were $\left(\mu_{\mathrm{o}} / \mu_{\mathrm{w}}=29.6, \rho_{\mathrm{o}} / \rho_{\mathrm{w}}=0.85\right.$, and $\sigma$ $=0.036 \mathrm{~N} / \mathrm{m}$ at $25.5^{\circ} \mathrm{C}$ ). They divided the six flow patterns observed into two groups, which are (i) segregated flow (ST, ST \& MI) and (ii) dispersed flow (Do/w \& w, o/w, Dw/o \& Do/w, w/o).

Vedapuri et al. [36] did a detailed study of the oil-water distribution across the pipe cross-section from top to bottom. They used two oils with viscosities of 2 and $96 \mathrm{mPa}$ s in a set-up which could be inclined at $0^{\circ}$ or $\pm 2^{\circ}$ with $101.2 \mathrm{~mm}$ ID pipe. The liquid velocities used in the experiment for the oil viscosities 2 and $96 \mathrm{mPa} s$ ranged from $0.4-1.6 \mathrm{~m} / \mathrm{s}$ and $0.4-0.8 \mathrm{~m} / \mathrm{s}$, respectively, with the water fraction of $20 \%, 40 \%, 60 \%$ and $80 \%$. They observed three flow patterns for the oil with a viscosity of $2 \mathrm{mPa} \cdot \mathrm{s}$ in horizontal inclination, which were semi-segregated, semi-mixed and semi-dispersed (homogeneous). They measured the liquid fractions along the vertical diameter of the pipe and plotted the thickness of the water layer to the diameter of the pipe $(\mathrm{h} / \mathrm{D})$ against the percentage of water.

Another popular method of constructing a flow map is to use the superficial water velocity $\left(\mathrm{v}_{\mathrm{sw}}\right)$ versus superficial oil velocity $\left(\mathrm{v}_{\mathrm{so}}\right)[11,22,29,41,56-58]$. The same flow patterns were identified by Al-Wahaibi et al. [22] and Yusuf et al. [29] because their experiments used the same fluids, operating conditions, and experimental set-up. The only difference being the diameter of the pipe used, which was 19 and $25.4 \mathrm{~mm}$ ID for Al-Wahaibi et al. [22] and Yusuf et al. [29], respectively. Their results are presented in Table 3.

Lovick and Angeli [55] focused on the study of dual continuous flow patterns (i.e., both phases maintain their continuity at the top and bottom of the pipe with inter-dispersion) in a $38 \mathrm{~mm} \mathrm{ID,}$ horizontal steel pipe. Water and EXXSOL D140 oil ( $\mu_{\mathrm{o}}=6 \mathrm{mPa} \cdot \mathrm{s}, \rho_{\mathrm{o}}=828 \mathrm{~kg} / \mathrm{m}^{3}$, interfacial tension $39.6 \mathrm{mN} / \mathrm{m}$ at $25^{\circ} \mathrm{C}$ ) were used as the test fluids. The fluids were brought together at the beginning of the test section via a modified T-junction, which minimises mixing. Several techniques were used to identify the flow patterns, as one technique not sufficient for all the flow patterns. The following flow patterns were identified: stratified wavy (SW), dual continuous flow (DC), dispersion of water-in-oil (Dw/o), and dispersion of oil-in-water (Do/w). They constructed a flow pattern map by plotting the mixture velocity $\left(\mathrm{v}_{\mathrm{m}}\right)$ against the input oil fraction $\left(\mathrm{f}_{\mathrm{o}}\right)$. They concluded that dual continuous flow appeared between the stratified and dispersed flow patterns at the intermediate mixture velocities for a range of input oil fractions. They also concluded that, during the dual continuous flow, as the oil input fraction increased, the velocity ratio also increased from less than 1 to greater than 1 .

Ibarra et al. [5] characterised the co-current flow of immiscible liquids with a dimensionless flow pattern map constructed from the ratio of mixture Reynolds number to Eötvös number $\left(\mathrm{Re}_{\mathrm{m}} / \mathrm{Eo}\right)$ as a function of water fraction $\left(f_{W}\right)$. The Reynolds number gives insight into the velocity field of a flow system: if the flow is laminar, viscous forces dominate, and the stratified liquid-liquid flow pattern appears [5]. If the flow is turbulent, there are instabilities at the interface, which promotes mixing and causes inertial forces to dominate and a transition to non-stratified flow [5]. At a fixed water fraction, an increase in the ratio of $\mathrm{Re}_{\mathrm{m}} /$ Eo led to the transition from separated flows to dispersed flow. Dispersion of oil-in-water with a water layer was only observed at high water fraction and $\mathrm{Re}_{\mathrm{m}} / \mathrm{Eo}$ between 200 and 800 . At low water fraction $\left(f_{w}<0.1\right)$, the stratified flow with droplets at the interface was observed. At these conditions, increasing $\mathrm{Re}_{\mathrm{m}} / \mathrm{Eo}$ led to the transition from stratified to dual continuous flow [5]. 
Brauner [2] proposed the use of a modified Eötvös number $\left(\mathrm{Eo}^{\prime}=\mathrm{Eo} / 8\right)$ for characterising oil-water flow in horizontal pipelines, which is Eötvös number (Eo) in Equation (1) with a factor of 8, taken from the Young-Laplace equation analysis for predicting the interface shape in stratified flow.

$$
\text { Eo }=\frac{\Delta \rho \mathrm{g} \mathrm{d}^{2}}{\sigma}
$$

A preliminary classification system for liquid-liquid flows by Brauner [2] is whether the Eo' $>>1$

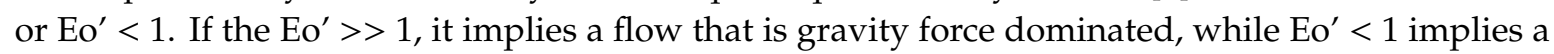
flow that is interfacial tension force dominated, where the Core annular flow is the most likely natural configuration [61]. In the gravitational force dominated flow, the stratified flow system tends to be the most likely natural configuration. Generally, the Eo' $>>1$ flow systems correspond to liquids with a finite density difference $(\Delta \rho)$ and sufficiently large pipe diameters where the interface can be assumed to be flat $[2,10]$.

Shi and Yeung [61] showed the absence of viscosity in the characterisation of liquid-liquid flow in horizontal pipes done by Brauner [2] using the Eötvös number. Therefore, they proposed the ratio of the gravitational force to viscous force $(\mathrm{G} / \mathrm{V})$ which includes the effect of viscosity in the configuration of the liquid-liquid flows, as presented in Equation (2). The gravity force to viscous force ratio can be expressed as:

$$
\frac{\mathrm{G}}{\mathrm{V}}=\frac{\Delta \rho \mathrm{g} \mathrm{d}^{2}}{\mu_{\mathrm{m}} \mathrm{v}_{\mathrm{m}}}
$$

where $\Delta \rho, g, d, \mu_{m}$ and $v_{m}$ are the difference in density of the two liquids, the acceleration due to gravity, the inner diameter of the pipe, mixture viscosity of the fluids, and mixture velocity of the liquids, respectively. The G/V ratio produces the completive role of gravity and viscous forces on the oil-water flows phase configuration.

\subsection{Harmonising the Major Flow Patterns in the Liquid-Liquid Horizontal Flow Literature}

Due to different names and terminologies used by various investigators to describe each of the flow patterns, it is difficult to combine or compare flow patterns. For simplicity and standardisation, flow patterns from different investigators that share common features are grouped and classified into slug flow, stratified flow, stratified mixed flow, dispersed oil-in-water flow, dispersed water-in-oil flow, and annular flow as shown in Figure 5. The flow patterns by Trallero et al. [11] is one of the most adopted flow patterns among researchers, but it fails to report slug and annular flow patterns. Annular flow tends to occur easily when oil viscosity is high, with a critical value of $35 \mathrm{mPa}$ s [15,54], or if the oil density is close to the water density $[7,15,42]$, or if the pipe diameter is small $[15,34,64]$. Unlike the gas-liquid flow patterns, which are sensitive to pipe diameters, the liquid-liquid flow patterns are more susceptible to the properties of the liquids such as interfacial tension, density, and viscosity [15]. Usually, for small diameter pipes (10 $\mathrm{mm}$ and smaller), different flow patterns were observed, such as the plug, slug, churn, and rivulet [64]. 


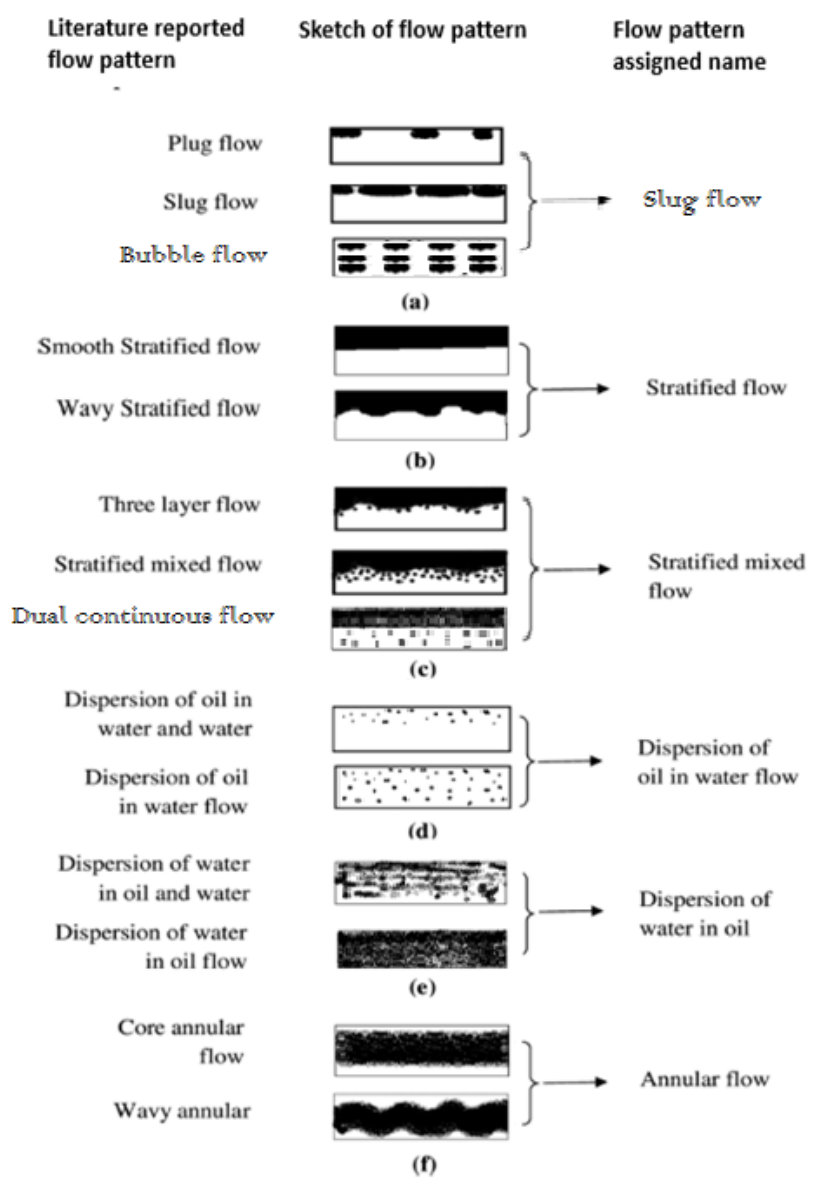

Figure 5. Grouping of the flow patterns modified after Dasari et al. [58].

\section{Dimensionless Parameter Definition}

There are three main approaches to defining the dimensionless groups characterising a liquid-liquid flow system [65]. These include dimensionless analysis, dynamic and kinematic ratio analysis, and basic equation analysis [65]. For dimensionless analysis, the Buckingham $\Pi$ theorem can be applied to the independent system variables and fundamental dimensions of the oil-water flow to determine the governing dimensionless groups [5]. For a fully developed, steady-state, isothermal oil-water flow in a horizontal pipeline, there are eleven relevant independent variables [5]. These include superficial oil and water velocities $\left(\mathrm{v}_{\mathrm{so}}\right)$ and $\left(\mathrm{v}_{\mathrm{sw}}\right)$, oil and water densities $\left(\rho_{\mathrm{o}}\right)$, and $\left(\rho_{\mathrm{w}}\right)$, pipe diameter $(\mathrm{d})$, oil and water viscosities $\left(\mu_{\mathrm{o}}\right)$ and $\left(\mu_{\mathrm{W}}\right)$, interfacial tension $(\sigma)$, pipe roughness $(\varepsilon)$, the acceleration due to gravity $(\mathrm{g})$, and wettability $(\alpha)$ (assumption: for horizontal orientation neglect inclination angle $(\theta)$ ). While the three fundamental dimensions are mass $(\mathrm{M})$, length $(\mathrm{L})$ and time $(\mathrm{T})$. Based on Buckingham $\Pi$ theorem, the number of system variables minus the number of fundamental variables is equal to the number of dimensionless groups [65]. Hence, eight dimensionless groups are expected from oil-water flow system, which Ibarra et al. [5] gave as (i) wettability, (ii) relative roughness, (iii) density ratio, (iv) viscosity ratio, (v) water fraction, (vi) mixture Reynold number, (vii) Eötvös number and (viii) mixture Froude number. However, Langhaar [66] and Shoham [65] stated that the formed dimensionless groups could be recombined to produce other groups not formed from the dimensionless analysis; in other words, by recombining the results of the dimensional analysis, one could generate an infinite/innumerable number of groups.

Plotting the superficial water velocity $\left(v_{\mathrm{sw}}=\mathrm{Q}_{\mathrm{w}} / \mathrm{A}\right)$ versus the superficial oil velocity $\left(\mathrm{v}_{\mathrm{so}}=\mathrm{Q}_{\mathrm{o}} / \mathrm{A}\right)$ is a common way of constructing flow pattern maps, where $\mathrm{A}$ is the cross-sectional area of the pipe and $\mathrm{Q}_{\mathrm{o}}$ and $\mathrm{Q}_{\mathrm{w}}$ are the volumetric flow rates of oil and water, respectively [10]. A flow pattern map for a two-phase flow system depends on the geometrical variables (pipe diameter and inclination angle), 
the fluid physical properties (densities, viscosities and surface tension) and operational variables (phase flow rates) $[19,25]$. These types of flow pattern maps are dimensional in that they are usually valid only for the particular pipe sizes and fluids employed to construct the flow maps [4]. Construction of flow pattern maps based on some relevant dimensionless groups would enable the possibility to compare and combine different experimental data and to develop more generalised flow pattern maps, which would cover a wide range of flow systems [5].

Some dimensionless groups governing the liquid-liquid flow are the ratio of some forces acting on the flow system. The following forces (inertia, viscous, gravity (buoyancy) and interfacial tension (capillary)) influence the liquid-liquid flow in horizontal pipelines [5]. Therefore, the effect of liquid properties (density, viscosity, and interfacial tension), pipe diameter and velocity are all included in the dimensionless parameters [5]. The ratio of the gravity force to the surface or interfacial tension force is expressed by the Eötvös number (Eo), presented in Equation (1). The Eötvös number (Eo) could predict the force that would be dominant in a liquid-liquid flow horizontal pipeline, based on the fluids properties and the internal pipe diameter.

\subsection{Mixture Reynolds Number to Eötvös number $\left(R_{m} / E o\right)$}

The Reynolds number (Re) is the ratio of the inertia force to viscous force in a flow system, while the mixture Reynolds number $\left(\operatorname{Re}_{\mathrm{m}}\right)$ can be expressed as

$$
\operatorname{Re}_{\mathrm{m}}=\frac{\rho_{\mathrm{m}} \mathrm{v}_{\mathrm{m}} \mathrm{d}}{\mu_{\mathrm{m}}}
$$

To calculate $\mathrm{Re}_{\mathrm{m}}$ from the homogeneous flow model, one must treat a liquid-liquid flow as a pseudo-single phase liquid with average liquid properties and average velocity [65]. Hence, in determining the thermal and fluid-dynamics behaviours of the fluid, no interface exchange is considered and only one mixture velocity, one mixture pressure and one mixture temperature are used $[67,68]$. The mixture velocity $\left(v_{m}\right)$, mixture density $\left(\rho_{m}\right)$, and mixture viscosity $\left(\mu_{m}\right)$ can be expressed in terms of volume fraction or mass fraction assuming no-slip conditions $[5,65,69]$.

$$
\begin{gathered}
v_{\mathrm{m}}=\frac{Q_{\mathrm{w}}+Q_{\mathrm{o}}}{A}=v_{\mathrm{sw}}+\mathrm{v}_{\mathrm{so}}, \\
\rho_{\mathrm{m}}=\rho_{\mathrm{w}} \mathrm{f}_{\mathrm{w}}+\rho_{\mathrm{o}}\left(1-\mathrm{f}_{\mathrm{w}}\right), \\
\mu_{\mathrm{m}}=\mu_{\mathrm{w}} \mathrm{f}_{\mathrm{w}}+\mu_{\mathrm{o}}\left(1-\mathrm{f}_{\mathrm{w}}\right)
\end{gathered}
$$

where $\mathrm{f}_{\mathrm{w}}$ is the water fraction, calculated as

$$
\mathrm{f}_{\mathrm{w}}=\frac{\mathrm{Q}_{\mathrm{w}}}{\mathrm{Q}_{\mathrm{w}+} \mathrm{Q}_{\mathrm{o}}}=\frac{\mathrm{v}_{\mathrm{sw}}}{\mathrm{v}_{\mathrm{sw}}+\mathrm{v}_{\mathrm{sw}}}
$$

Then, the ratio can be expressed as

$$
\frac{\operatorname{Re}_{\mathrm{m}}}{\mathrm{Eo}}=\frac{\rho_{\mathrm{m}} \mathrm{v}_{\mathrm{m}}}{\mu_{\mathrm{m}}} \frac{\sigma}{\Delta \rho \mathrm{gd}}
$$

The ratio of mixture Reynolds number to Eötvös number $\mathrm{Re}_{\mathrm{m}} / \mathrm{Eo}$ as a function of water fraction $\left(f_{\mathrm{w}}\right)$ has been applied by Ibarra et al. [5] to create a dimensionless flow pattern transitions map.

\subsection{Weber Number to Eötvös Number (We/Eo)}

The Weber number is the ratio of inertia force to the interfacial tension force, while the ratio of the difference in the two-phase gravity forces to interfacial tension force is called the Eötvös number. 
Therefore, the We/Eo can be expressed as the ratio of inertia force to the difference in the two-phase gravity forces.

$$
\frac{\mathrm{We}}{\mathrm{Eo}}=\frac{\rho_{\mathrm{m}} \mathrm{v}_{\mathrm{m}}^{2}}{\Delta \rho \mathrm{g} \mathrm{d}}
$$

A high We/Eo depicts the dominance of inertia force over buoyancy, where the flow will be turbulent, and there will be instabilities at the interface between the two liquids. The dispersed flows are the most likely flow patterns in this region. At low We/Eo, the gravity forces dominate, hence the gravitational effect will make the dense liquid to flow at the bottom of the pipe while the light liquid flows at the top. The most likely flow pattern is stratified flow.

\subsection{Mixture Froude Number $\left(F r_{m}\right)$, Oil Froude Number $\left(F r_{o}\right)$ and Water Froude Number $\left(F r_{w}\right)$}

The mixture Froude number $\left(\mathrm{Fr}_{\mathrm{m}}\right)$ is the ratio of inertia force to the gravity force based on the average properties of the two immiscible fluids (oil and water) as explained by the mixture Reynolds number to Eötvös number $\left(\mathrm{Re}_{\mathrm{m}} / \mathrm{Eo}\right)$.

$$
\begin{aligned}
& \operatorname{Fr}_{\mathrm{m}}^{2}=\frac{\mathrm{v}_{\mathrm{m}}^{2}}{\mathrm{gd}} \frac{\rho_{\mathrm{m}}}{\left(\rho_{\mathrm{w}}-\rho_{\mathrm{o}}\right)} \\
& \mathrm{Fr}_{\mathrm{O}}^{2}=\frac{\mathrm{v}_{\mathrm{O}}^{2}}{\mathrm{gd}} \frac{\rho_{\mathrm{O}}}{\left(\rho_{\mathrm{w}}-\rho_{\mathrm{o}}\right)} \\
& \mathrm{Fr}_{\mathrm{W}}^{2}=\frac{\mathrm{v}_{\mathrm{W}}^{2}}{\mathrm{gd}} \frac{\rho_{\mathrm{W}}}{\left(\rho_{\mathrm{w}}-\rho_{\mathrm{o}}\right)}
\end{aligned}
$$

By definition, both We/Eo and $\mathrm{Fr}_{\mathrm{m}}$ are ratios of inertia force to the gravity force so that one can determine the relationship between them.

Divide We/Eo by $\mathrm{Fr}_{\mathrm{m}}$

$$
\frac{\frac{W e}{E o}}{\operatorname{Fr}_{m}^{2}}=\frac{\frac{\rho_{\mathrm{m}} v_{m}^{2}}{\Delta \rho \mathrm{gd}}}{\frac{v_{m}^{2}}{\mathrm{gd}} \frac{\rho_{\mathrm{m}}}{\left(\rho_{\mathrm{w}}-\rho_{\mathrm{o}}\right)}}=1
$$

Hence,

$$
\mathrm{Fr}_{\mathrm{m}}=\left(\frac{\mathrm{We}}{\mathrm{Eo}}\right)^{1 / 2}
$$

The turbulence of We/Eo will be the square of the turbulence of $\mathrm{Fr}_{\mathrm{m}}$ when the inertia force is dominant. Dispersed flow patterns are more likely in this region. Where gravity force is dominant, the gravitational effect will be high, which will cause the stratification of the oil and water flow, and the value of $\mathrm{Fr}_{\mathrm{m}}$ will be a square root of the We/Eo value. Both $\mathrm{Fr}_{\mathrm{m}}$ and We/Eo give the same information but at different magnitudes.

\subsection{Gravity Force to Viscous Force $(G / V)$}

The ratio of gravity and viscous forces for the oil-water flows is represented as G/V [61]. At high G/V in oil-water flows, the gravity force dominates, while the viscous force is comparatively low (i.e., low oil viscosity, since water viscosity is constant). For this case, the fluid will break up easily at high kinetic energy, and the most likely flow structures are stratified flow and fine dispersions of one of the liquids in the continuum of the other [61]. At low G/V in oil-water flow, the stratified flow is less likely to form because the gravitational force effect is comparatively small. The probable flow configurations are oil core flowing inside annular water: continuously to generate annular (core annular flow) or discontinuously to produce intermittent flows (slug, plug) in which shear stress is minimal [61]. With high turbulence kinetic energy flow, the higher viscous (oil) phase is too viscous to be broken by the kinetic energy into fine drops but lumps of various sizes and shapes. For a liquid-liquid flow with 
medium G/V, both the gravity and viscous forces influence this region, and all the flow patterns can occur within the region.

\section{Results}

\subsection{Data Sources}

Data gathering and analysis are presented. All the datasets used in developing some flow maps were obtained from liquid-liquid experimental works published in scholarly journals. The density of oil experimented in all the cases is less than water density, and all the considered experimental works were performed in the horizontal orientation. Brief details of each of the experimental works are presented in Table 4.

Table 4. Sources of the gathered datasets.

\begin{tabular}{|c|c|c|c|}
\hline \multicolumn{2}{|c|}{ Trallero et al. [11] } & \multicolumn{2}{|c|}{ Soleimani [52] } \\
\hline Tap water density $\left(\mathrm{kg} / \mathrm{m}^{3}\right)$ & $997 *$ & Tap water density $\left(\mathrm{kg} / \mathrm{m}^{3}\right)$ & 998 \\
\hline White oil density $\left(\mathrm{kg} / \mathrm{m}^{3}\right)$ & 884 & Exxsol 80 density $\left(\mathrm{kg} / \mathrm{m}^{3}\right)$ & 801 \\
\hline Tap water viscosity (Pa s) & 0.00097 & Tap water viscosity (Pa s) & 0.001 \\
\hline White oil viscosity (Pa s) & 0.0288 & Exxsol 80 viscosity (Pa s) & 0.0016 \\
\hline Interfacial tension $(\mathrm{N} / \mathrm{m})$ & 0.036 & Interfacial tension $(\mathrm{N} / \mathrm{m})$ & $0.017 @ 22{ }^{\circ} \mathrm{C}$ \\
\hline Diameter $(\mathrm{m})$ & 0.0501 & Diameter $(\mathrm{m})$ & 0.0243 \\
\hline Temperature $\left({ }^{\circ} \mathrm{C}\right)$ & 25.5 & Temperature $\left({ }^{\circ} \mathrm{C}\right)$ & 25 \\
\hline \multicolumn{2}{|c|}{ Al-Wahaibi et al. [22] } & \multicolumn{2}{|c|}{ Vielma et al. [57] } \\
\hline Water density $\left(\mathrm{kg} / \mathrm{m}^{3}\right)$ & 998 & Tap water density $\left(\mathrm{kg} / \mathrm{m}^{3}\right)$ & 998 \\
\hline Mineral oil density $\left(\mathrm{kg} / \mathrm{m}^{3}\right)$ & 875 & Mineral oil density $\left(\mathrm{kg} / \mathrm{m}^{3}\right)$ & $860 * *$ \\
\hline Water viscosity (Pa s) & 0.001 & Tap water viscosity (Pa s) & 0.001 \\
\hline Mineral oil viscosity (Pa s) & 0.012 & Mineral oil viscosity (Pa s) & $0.0188^{* *}$ \\
\hline Interfacial tension $(\mathrm{N} / \mathrm{m})$ & 0.0201 & Interfacial tension $(\mathrm{N} / \mathrm{m})$ & $0.0285^{* *}$ \\
\hline Diameter $(\mathrm{m})$ & 0.019 & Diameter $(\mathrm{m})$ & 0.0508 \\
\hline Temperature $\left({ }^{\circ} \mathrm{C}\right)$ & N/A & Temperature $\left({ }^{\circ} \mathrm{C}\right)$ & 25.5 \\
\hline \multicolumn{2}{|c|}{ Dasari et al. [58] } & \multicolumn{2}{|c|}{ Guzhov et al. [38] } \\
\hline Water density $\left(\mathrm{kg} / \mathrm{m}^{3}\right)$ & 997 & Tap water density $\left(\mathrm{kg} / \mathrm{m}^{3}\right)$ & 998 \\
\hline Lube oil density $\left(\mathrm{kg} / \mathrm{m}^{3}\right)$ & 889 & Mineral oil density $\left(\mathrm{kg} / \mathrm{m}^{3}\right)$ & 896 \\
\hline Water viscosity (Pa s) & 0.001 & Tap water viscosity (Pa s) & 0.001 \\
\hline Lube oil viscosity (Pa s) & 0.107 & Mineral oil viscosity (Pa s) & 0.0218 \\
\hline Interfacial tension $(\mathrm{N} / \mathrm{m})$ & 0.024 & Interfacial tension $(\mathrm{N} / \mathrm{m})$ & 0.0448 \\
\hline Diameter $(\mathrm{m})$ & 0.025 & Diameter $(\mathrm{m})$ & 0.0394 \\
\hline Temperature $\left({ }^{\circ} \mathrm{C}\right)$ & 25 & Temperature $\left({ }^{\circ} \mathrm{C}\right)$ & 21 \\
\hline \multicolumn{2}{|c|}{ Grassi et al. [41] } & \multicolumn{2}{|c|}{ Tan et al. [3] 20\# } \\
\hline Water density $\left(\mathrm{kg} / \mathrm{m}^{3}\right)$ & 997 & Tap water density $\left(\mathrm{kg} / \mathrm{m}^{3}\right)$ & 998 \\
\hline Oil density $\left(\mathrm{kg} / \mathrm{m}^{3}\right)$ & 886 & Mineral oil density $\left(\mathrm{kg} / \mathrm{m}^{3}\right)$ & 888 \\
\hline Water viscosity (Pa s) & 0.0013 & Tap water viscosity (Pa s) & 0.001 \\
\hline Oil viscosity (Pa s) & 0.653 & Mineral oil viscosity (Pa s) & 0.02 \\
\hline Interfacial tension $(\mathrm{N} / \mathrm{m})$ & 0.05 & Interfacial tension $(\mathrm{N} / \mathrm{m})$ & 0.01897 \\
\hline Diameter $(\mathrm{m})$ & 0.021 & Diameter $(\mathrm{m})$ & 0.0145 \\
\hline Temperature $\left({ }^{\circ} \mathrm{C}\right)$ & 25 & Temperature $\left({ }^{\circ} \mathrm{C}\right)$ & 25 \\
\hline \multicolumn{2}{|c|}{ Chakrabarti et al. [56] } & \multicolumn{2}{|c|}{ Tan et al. [3] 200\# } \\
\hline Water density $\left(\mathrm{kg} / \mathrm{m}^{3}\right)$ & 997 & Tap water density $\left(\mathrm{kg} / \mathrm{m}^{3}\right)$ & 998 \\
\hline Kerosine $\left(\mathrm{kg} / \mathrm{m}^{3}\right)$ & 787 & Mineral oil density $\left(\mathrm{kg} / \mathrm{m}^{3}\right)$ & 869 \\
\hline Water viscosity (Pa s) & 0.00084 & Tap water viscosity (Pa s) & 0.001 \\
\hline Kerosine (Pa s) & 0.0012 & Mineral oil viscosity (Pa s) & 0.237 \\
\hline Interfacial tension $(\mathrm{N} / \mathrm{m})$ & 0.045 & Interfacial tension $(\mathrm{N} / \mathrm{m})$ & 0.04583 \\
\hline Diameter $(\mathrm{m})$ & 0.0254 & Diameter $(\mathrm{m})$ & 0.0145 \\
\hline Temperature $\left({ }^{\circ} \mathrm{C}\right)$ & 25 & Temperature $\left({ }^{\circ} \mathrm{C}\right)$ & 25 \\
\hline
\end{tabular}


Table 4. Cont.

\begin{tabular}{cccc}
\hline Lovick and Angeli [55] & \multicolumn{2}{c}{ Tan et al. [3] 400\# } \\
\hline Tap water density $\left(\mathrm{kg} / \mathrm{m}^{3}\right)$ & 998 & Tap water density $\left(\mathrm{kg} / \mathrm{m}^{3}\right)$ & 998 \\
Exxsol140 density $\left(\mathrm{kg} / \mathrm{m}^{3}\right)$ & 828 & Mineral oil density $\left(\mathrm{kg} / \mathrm{m}^{3}\right)$ & 896 \\
Tap water viscosity $(\mathrm{Pa} \mathrm{s})$ & 0.001 & Tap water viscosity $(\mathrm{Pa} \mathrm{s})$ & 0.001 \\
Exxsol140 viscosity $(\mathrm{Pa} \mathrm{s})$ & 0.006 & Mineral oil viscosity $(\mathrm{Pa} \mathrm{s})$ & 0.456 \\
Interfacial tension $(\mathrm{N} / \mathrm{m})$ & 0.0396 & Interfacial tension $(\mathrm{N} / \mathrm{m})$ & 0.05149 \\
Diameter $(\mathrm{m})$ & 0.038 & Diameter $(\mathrm{m})$ & 0.0145 \\
Temperature $\left({ }^{\circ} \mathrm{C}\right)$ & 25 & Temperature $\left({ }^{\circ} \mathrm{C}\right)$ & 25
\end{tabular}

Note: for data with ${ }^{(*)}$, the authors recorded $1037 \mathrm{~kg} / \mathrm{m}^{3}$ for tap water at $\approx 25.5^{\circ} \mathrm{C}$. For data with ${ }^{(* *)}$, the authors compared these values with Trallero et al. [11] data; hence those values are considered to be at the same temperature with Trallero et al. [11] data.

Based on similar operating conditions for the considered experimental studies, water properties are quite similar, while oil density and oil viscosity range between $787-896 \mathrm{~kg} / \mathrm{m}^{3}$ and $0.006-0.653 \mathrm{~Pa} \mathrm{~s}$, respectively. The pipe diameter ranges between $0.0145-0.0508 \mathrm{~m}$, while the interfacial tension ranges between $0.017-0.05149 \mathrm{~N} / \mathrm{m}$. Each source contributed datapoints to the datasets as follows: Trallero et al. [11] = 251, Soleimani [52] = 166, Al-Wahaibi et al. [22] = 196, Vielma et al. [57] = 153, Dasari et al. [58] $=538$, Guzhov et al. [38] = 176, Grassi et al. [41] = 137, Tan et al. [3] 20\# = 112, Chakrabarti et al. [56] = 506, Tan et al. [3] 200\# = 224, Lovick and Angeli [55] = 114, and Tan et al. [3] $400 \#=123$.

\subsection{Flow Pattern Maps Construction}

Figure 6 presents a flow pattern map of the ratio of mixture Reynold number to Eötvös number $\left(\mathrm{Re}_{\mathrm{m}} / \mathrm{Eo}\right)$ versus water fraction $\left(\mathrm{f}_{\mathrm{w}}\right)$, a representation of one of various combinations of dimensionless groups under consideration. The data were further classified based on oil viscosity and diameter to see if the data from a particular flow pattern would form a better cluster on the flow map. The results are discussed below.

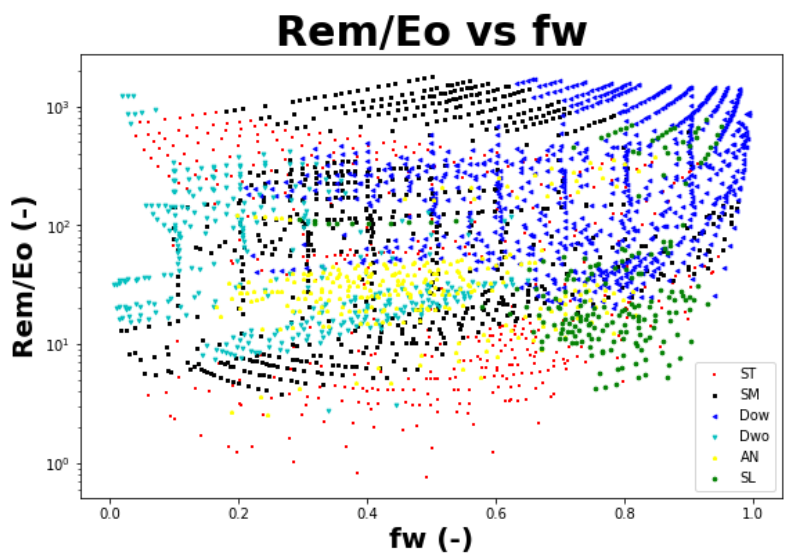

Figure 6. The ratio of mixture Reynold number to Eötvös number $\left(\operatorname{Re}_{\mathrm{m}} / \mathrm{Eo}\right)$ versus water fraction $\left(\mathrm{f}_{\mathrm{w}}\right)$ for the gathered published experimental datasets. Note: data from $[3,11,22,38,41,52,55-58]$. Data points denoted with red squares, black rectangles, blue triangle_left, cyan triangle_down, yellow circle and green star represent stratified flow (ST), stratified mixed flow (SM), dispersed oil-in-water flow (Do/w), dispersed water-in-oil flow (Dw/o), annular flow (AN) and slug flow (SL), respectively.

\subsubsection{Flow Pattern Maps Based on Oil Viscosity}

Abubakar et al. [45] and Tan et al. [3] classified oil viscosity into $<10 \mathrm{mPa}$ s, 20-100 $\mathrm{mPa} \mathrm{s}$ and $>100 \mathrm{mPa}$ s as low-viscous oil (LVO), medium-viscous oil (MVO) and heavy-viscous oil (HVO), respectively. Figure 7 presents flow maps of the ratio of mixture Reynold number to Eötvös number $\left(\operatorname{Re}_{\mathrm{m}} / \mathrm{Eo}\right)$ versus water fraction $\left(\mathrm{f}_{\mathrm{W}}\right)$ after regrouping of data by oil viscosity. 
(a)

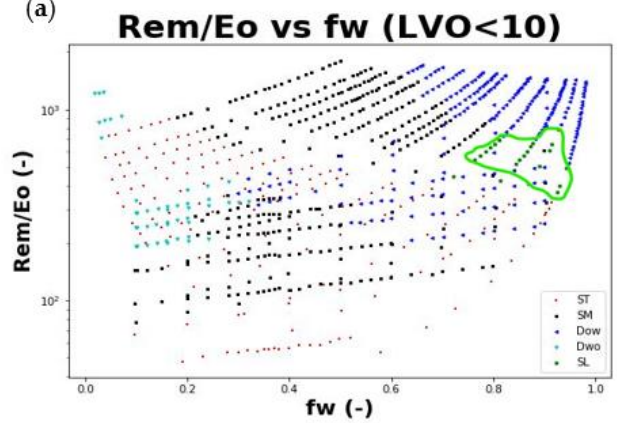

(c)

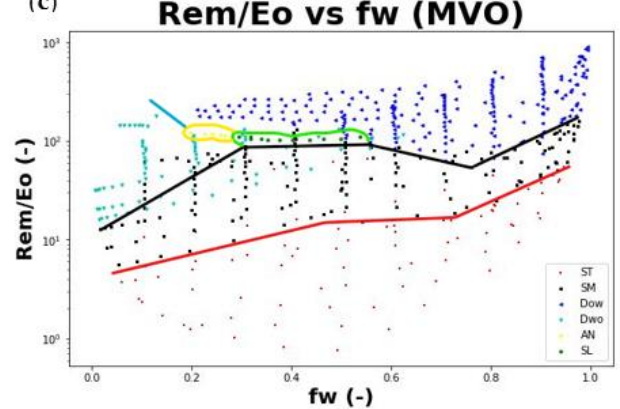

(b) $\quad$ Rem/Eo vs fw $($ LVO $<20)$

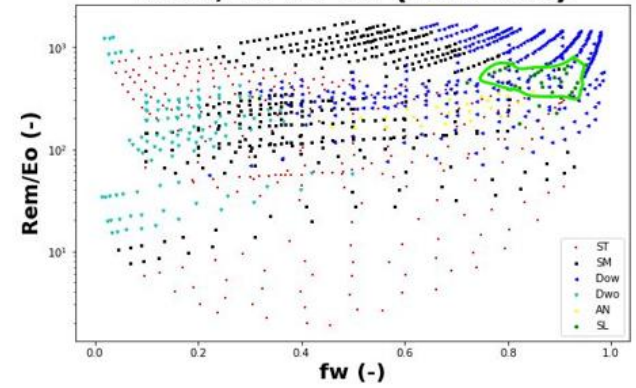

(d)

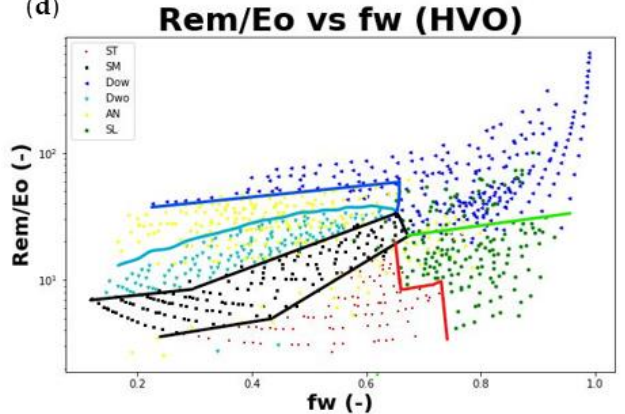

Figure 7. The ratio mixture Reynolds number to Eötvös number $\left(\operatorname{Re}_{\mathrm{m}} / \mathrm{Eo}\right)$ versus water fraction $\left(\mathrm{f}_{\mathrm{W}}\right),(\mathbf{a})<10 \mathrm{mPa} \mathrm{s}(\mathbf{b})<20 \mathrm{mPa} \mathrm{s}$ (c) $20-100 \mathrm{mPa} \cdot \mathrm{s},(\mathbf{d})>100 \mathrm{mPa} \mathrm{s}$, for the gathered published experimental datasets. Note: data points denoted with red squares, black rectangles, blue triangle_left, cyan triangle_down, yellow circle and green star represent stratified flow (ST), stratified mixed flow (SM), dispersed oil-in-water flow (Do/w), dispersed water-in-oil flow (Dw/o), annular flow (AN) and slug flow (SL), respectively. Each boundary line denoted with red, black, blue, cyan, yellow and green encloses-partially or completely-stratified flow (ST), stratified mixed flow (SM), dispersed oil-in-water flow (Do/w), dispersed water-in-oil flow (Dw/o), annular flow (AN) and slug flow $(\mathrm{SL})$, respectively.

The oil viscosity between $10-20 \mathrm{mPa} s$ was missing in the classification, so another class of $<20 \mathrm{mPa}$ s was considered as only two of the selected experimental works have their oil viscosity in the range of 10-20 mPa s. The same analysis was performed using the other combination of dimensionless groups, and the results are presented in Table 5.

Table 5. A summary of constructed flow pattern maps using dimensionless parameters.

\begin{tabular}{|c|c|c|c|c|c|c|}
\hline & ST & SM & Do/w & Dw/o & SL & AN \\
\hline $\operatorname{Re}_{\mathrm{m}} /$ Eo versus $\mathrm{f}_{\mathrm{W}}$ & $\mathrm{N}$ & $\mathrm{N}$ & $\mathrm{N}$ & $\mathrm{N}$ & $\mathrm{N}$ & $\mathrm{N}$ \\
\hline $\operatorname{Re}_{\mathrm{m}} /$ Eo versus $\mathrm{f}_{\mathrm{w}}(\mathrm{LVO}<10)$ & $\mathrm{N}$ & $\mathrm{N}$ & $\mathrm{N}$ & $\mathrm{N}$ & $\mathrm{Y}$ & N/A \\
\hline $\operatorname{Re}_{\mathrm{m}} /$ Eo versus $\mathrm{f}_{\mathrm{w}}(\mathrm{LVO}<20)$ & $\mathrm{N}$ & $\mathrm{N}$ & $\mathrm{N}$ & $\mathrm{N}$ & $\mathrm{Y}$ & $\mathrm{N}$ \\
\hline $\operatorname{Re}_{\mathrm{m}} /$ Eo versus $\mathrm{f}_{\mathrm{w}}(\mathrm{MVO})$ & $\mathrm{Y}$ & Y & Y & Y & $Y^{*}$ & $\mathrm{Y}^{*}$ \\
\hline $\operatorname{Re}_{\mathrm{m}} /$ Eo versus $\mathrm{f}_{\mathrm{w}}(\mathrm{HVO})$ & $\mathrm{Y}$ & Y & Y & Y & $\mathrm{Y}$ & $\mathrm{N}$ \\
\hline We/Eo versus $f_{w}$ & $\mathrm{Y}$ & Y & $\mathrm{N}$ & Y & $\mathrm{Y}$ & $\mathrm{N}$ \\
\hline $\mathrm{Fr}_{\mathrm{m}}$ versus $\mathrm{f}_{\mathrm{W}}$ & $\mathrm{Y}$ & Y & $\mathrm{N}$ & $\mathrm{Y}$ & $\mathrm{Y}$ & $\mathrm{N}$ \\
\hline$G / V$ versus $f_{W}$ & $\mathrm{~N}$ & $\mathrm{~N}$ & $\mathrm{~N}$ & $\mathrm{~N}$ & $\mathrm{~N}$ & $\mathrm{~N}$ \\
\hline$G / V$ versus $f_{w}(L V O<10)$ & Y & $\mathrm{Y}$ & Y & $\mathrm{Y}$ & Y & N/A \\
\hline $\mathrm{G} / \mathrm{V}$ versus $\mathrm{f}_{\mathrm{w}}(\mathrm{LVO}<20)$ & $\mathrm{N}$ & $\mathrm{N}$ & $\mathrm{N}$ & Y & $\mathrm{N}$ & $\mathrm{Y}$ \\
\hline$G / V$ versus $f_{w}(M V O)$ & $\mathrm{Y}$ & $\mathrm{N}$ & $\mathrm{N}$ & $\mathrm{N}$ & $\mathrm{Y}$ & $\mathrm{Y}$ \\
\hline$G / V$ versus $f_{w}(H V O)$ & $\mathrm{Y}$ & Y & $\mathrm{N}$ & $\mathrm{Y}$ & $\mathrm{N}$ & $\mathrm{Y}$ \\
\hline $\mathrm{Fr}_{\mathrm{w}}$ versus $\mathrm{Fr}_{\mathrm{O}}$ & $\mathrm{N}$ & $\mathrm{N}$ & $\mathrm{N}$ & $\mathrm{N}$ & $\mathrm{N}$ & $\mathrm{N}$ \\
\hline $\mathrm{Fr}_{\mathrm{w}}$ versus $\mathrm{Fr}_{\mathrm{O}}(\mathrm{LVO}<10)$ & Y & $\mathrm{Y}$ & Y & $\mathrm{Y}$ & $\mathrm{Y}$ & N/A \\
\hline $\mathrm{Fr}_{\mathrm{W}}$ versus $\mathrm{Fr}_{\mathrm{O}}(\mathrm{LVO}<20)$ & $\mathrm{N}$ & $\mathrm{N}$ & $\mathrm{N}$ & $\mathrm{N}$ & $\mathrm{N}$ & $\mathrm{N}$ \\
\hline $\mathrm{Fr}_{\mathrm{W}}$ versus $\mathrm{Fr}_{\mathrm{O}}(\mathrm{MVO})$ & Y & $\mathrm{Y}$ & Y & $\mathrm{Y}$ & $Y^{*}$ & $\mathrm{Y}^{*}$ \\
\hline $\mathrm{Fr}_{\mathrm{W}}$ versus $\mathrm{Fr}_{\mathrm{O}}(\mathrm{HVO})$ & $\mathrm{Y}$ & Y & $\mathrm{N}$ & Y & $\mathrm{N}$ & $\mathrm{N}$ \\
\hline
\end{tabular}

Note: * means a few data points, $\mathrm{Y}$ means yes, flow pattern marked out, $\mathrm{N}$ means no, flow pattern not marked out, N/A means not applicable. Note: ST means stratified flow, SM means stratified mixed flow, Do/w means dispersed oil-in-water flow, Dw/o means dispersed water-in-oil, SL means slug flow and AN means annular flow. 


\subsubsection{Flow Pattern Maps Based on the Pipe Diameter}

Cai et al. [15] showed that liquid-liquid flow patterns are less susceptible to pipe diameter, unlike gas-liquid flow patterns. However, it remains important to investigate the effect of pipe diameter on the flow patterns of liquid-liquid flows; this will be achieved by regrouping the data points based on the pipe diameter of the experimental set-up. Three classes of diameters considered, namely $\leq 20 \mathrm{~mm}$, 21-30 $\mathrm{mm}$ and $>30 \mathrm{~mm}$, which are referred to as class 1 , class 2 and class 3 , respectively.

The combinations of dimensionless groups used for the analysis are (i) $\operatorname{Re}_{m} / E$ Eo versus $f_{w}$, (ii) We/Eo versus $f_{w}$, (iii) $F_{m}$ versus $f_{w}$, (iv) $G / V$ versus $f_{w}$, $(v) F_{w}$ versus $F_{r}$. Let $F_{m}$ versus $f_{w}$ be a representative combination of dimensionless groups to show the effect of pipe diameters on the liquid-liquid flow pattern in the horizontal pipeline, as presented in Figure 8. The experimental works in class 1 are those of Al-Wahaibi et al. [22] and Tan et al. [3] 20\#, 200\# \& 400\# with diameters of 19 and $14.5 \mathrm{~mm}$, respectively. The experimental works in class 2 are taken from Chakrabarti et al. [56], Dasari et al. [58], Grassi et al. [41], and Soleimani [52] with diameters of 25.4, 25, 21, and $24.3 \mathrm{~mm}$, respectively. The experimental works in class 3 are those of Guzhov et al. [38], Lovick and Angeli [55], Trallero et al. [11], and Vielma et al. [57] with diameters of 39.4, 38, 50.1, and $50.8 \mathrm{~mm}$, respectively.
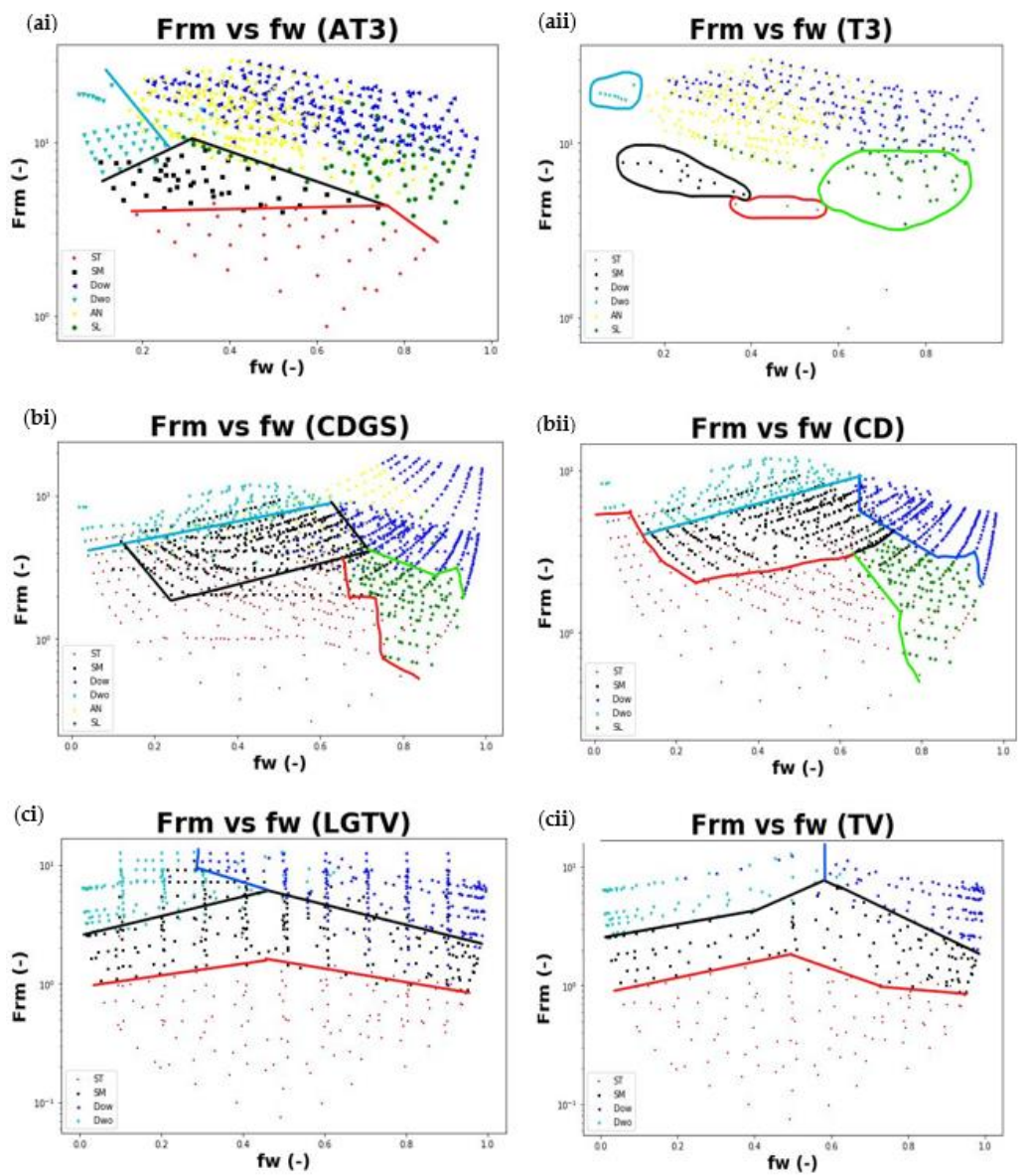

Figure 8. The mixture Froude number $\mathrm{Fr}_{\mathrm{m}}$ versus water fraction $\mathrm{f}_{\mathrm{w}}$, (ai) class 1 , (aii) class 1 with experimental work of a diameter $14.5 \mathrm{~mm}$, (bi) class 2, (bii) class 2 with experimental works of diameters 25 and $25.4 \mathrm{~mm}$, (ci) class 3, (cii) class 3 with experimental works of diameters 50.1 and $50.8 \mathrm{~mm}$, for the gathered published experimental datasets. Note: data points denoted with red squares, black rectangles, blue triangle_left, cyan triangle_down, yellow circle and green star represent stratified flow (ST), stratified mixed flow (SM), dispersed oil-in-water flow (Do/w), dispersed water-in-oil flow (Dw/o), annular flow (AN) and slug flow (SL), respectively. Each boundary line denotes with red, black, blue, cyan and green encloses-partially or completely—stratified flow (ST), stratified mixed flow (SM), dispersed oil-in-water flow (Do/w), dispersed water-in-oil flow (Dw/o) and slug flow (SL), respectively. 


\section{Discussion}

\subsection{Data Analysis}

The use of dimensionless groups is to normalise the data points of a particular flow pattern so that the data points can form a distinct region on a flow map. The gathered datasets are placed in six classes of flow patterns, which are stratified (ST), stratified mixed (SM), dispersed oil in water (Do/w), dispersed water in oil (Dw/o), annular (AN) and slug (SL) flows. There are several combinations of dimensionless groups that can be used for constructing a flow map and those considered in this work are: (i) the ratio of mixture Reynold number to Eötvös number $\left(\operatorname{Re}_{m} / E o\right)$ versus water fraction $\left(f_{W}\right)$, (ii) the ratio of Weber number to Eötvös number $(\mathrm{We} / \mathrm{Eo})$ versus water fraction $\left(\mathrm{f}_{\mathrm{W}}\right)$, (iii) the mixture Froude number $\left(\mathrm{Fr}_{\mathrm{m}}\right)$ versus water fraction $\left(f_{\mathrm{W}}\right)$, (iv) the ratio of gravity force to viscous force $\mathrm{G} / \mathrm{V}$ versus water fraction $\left(f_{w}\right),(v)$ the water Froude number $\left(\mathrm{Fr}_{\mathrm{w}}\right)$ versus the oil Froude number $\left(\mathrm{Fr}_{\mathrm{o}}\right)$.

The following combinations of dimensionless groups $\left(\operatorname{Re}_{m} /\right.$ Eo versus $_{\mathrm{w}}, \mathrm{G} / \mathrm{V}$ versus $\mathrm{f}_{\mathrm{w}}$, and $\mathrm{Fr}_{\mathrm{w}}$ versus $\mathrm{Fr}_{\mathrm{o}}$ ) show that all flow patterns form more than one trend on the flow pattern map. Hence, there is no distinct region on the flow pattern map that can be designated for a particular flow pattern. Figure 6 gives a representation of these combinations of dimensionless groups. The data were further classified based on oil viscosity and diameter to see if the data from a particular flow pattern would form a better cluster on the flow map. The results are discussed below.

\subsubsection{Data Analysis Based on Oil Viscosity}

After regrouping the gathered data by oil viscosity, a better trend of the flow patterns was formed on the flow map in Figure 7 as compared to Figure 6, but not all the flow patterns could be delineated on the flow map. The flow patterns were better marked out when the data points were classified based on their oil viscosity in all cases, as presented in Table 5. However, all the flow patterns cannot be marked out on a flow map using any of the considered dimensionless group combinations, even where the oil viscosity is one of the variables in the formation of the combined dimensionless groups i.e., (i) $\operatorname{Re}_{\mathrm{m}} /$ Eo versus $\mathrm{f}_{\mathrm{w}}$, (ii) $\mathrm{G} / \mathrm{V}$ versus $\mathrm{f}_{\mathrm{w}}$, the flow patterns were still not completely normalised. In low-viscous oil $(\mathrm{LVO})<20 \mathrm{mPa}$ s category, $\mathrm{Fr}_{\mathrm{w}}$ versus $\mathrm{Fr}_{\mathrm{o}}$ normalised no flow pattern, $\mathrm{Re}_{\mathrm{m}} / \mathrm{Eo}$ versus $\mathrm{f}_{\mathrm{w}}$ delineated only SL flow pattern, and $\mathrm{G} / \mathrm{V}$ versus $\mathrm{f}_{\mathrm{w}}$ normalised Dw/o and AN flow patterns. In low-viscous oil $(\mathrm{LVO})<10 \mathrm{mPa}$ s category, $\mathrm{Fr}_{\mathrm{w}}$ versus $\mathrm{Fr}_{\mathrm{o}}$ and $\mathrm{G} / \mathrm{V}$ versus $\mathrm{f}_{\mathrm{w}}$ normalised all the flow patterns available for the analysis, while $\mathrm{Re}_{\mathrm{m}} /$ Eo versus $\mathrm{f}_{\mathrm{w}}$ delineated only SL flow pattern. Hence, $(\mathrm{LVO})<20 \mathrm{mPa}$ s group may be too broad for the analysis.

\subsubsection{Data Analysis Based on the Pipe Diameter}

Figure 8ai contains experimental works with diameters 14.5 and $19 \mathrm{~mm}$, while Figure 8aii contains experimental works with a diameter of $14.5 \mathrm{~mm}$. The $\mathrm{Fr}_{\mathrm{m}}$ versus $\mathrm{f}_{\mathrm{w}}$ was expected to normalise the flow patterns, particularly as the diameter range was narrowed. However, the following flow patterns Dow, AN and SL, and Dow and AN could not be delineated in Figures 8ai and 8aii, respectively.

Likewise, to check the effect of a slight change in diameter, the experimental works with diameters of 21, 24.3, 25 and $25.4 \mathrm{~mm}$ were plotted Figure 8bi, whilst the data associated with slightly bigger pipe diameters of 25.4 and $25 \mathrm{~mm}$ are shown in Figure 8bii. With a wider diameter range (Figure 8bi), the dimensionless groups do not normalise all the flow patterns, but with a slight change in diameter (Figure 8bii) all the flow patterns were normalised and delineated.

Figure 8ci has the experimental works with diameters of 39.4, 38, 50.1 and $50.8 \mathrm{~mm}$, but Figure 8cii has a narrower diameter range of 50.1 and $50.8 \mathrm{~mm}$. In both cases, the $\mathrm{Fr}_{\mathrm{m}}$ versus $\mathrm{f}_{\mathrm{w}}$ normalised and delineated the flow patterns.

\section{Conclusions}

A literature review on experimental studies and development of flow pattern maps of liquid-liquid flow in horizontal pipes were conducted. Relevant datasets were obtained from twelve experimental 
studies published in scholarly journals. The following combinations of dimensionless groups ((i) the ratio of mixture Reynold number to Eötvös number $\left(\mathrm{Re}_{\mathrm{m}} / \mathrm{Eo}\right)$ versus water fraction $\left(\mathrm{f}_{\mathrm{w}}\right)$, (ii) the ratio of Weber number to Eötvös number (We/Eo) versus water fraction $\left(f_{W}\right)$, (iii) the mixture Froude number $\left(\mathrm{Fr}_{\mathrm{m}}\right)$ versus water fraction $\left(f_{\mathrm{w}}\right)$, (iv) the ratio of gravity force to viscous force $(\mathrm{G} / \mathrm{V})$ versus water fraction $\left(f_{w}\right),(v)$ the water Froude number $\left(\mathrm{Fr}_{\mathrm{W}}\right)$ versus the oil Froude number $\left.\left(\mathrm{Fr}_{\mathrm{o}}\right)\right)$ were used to obtain more generalised flow pattern maps.

- Based on the analysis done in this project, it can be concluded that each flow pattern formed more than one trend on the flow map when data from the twelve sources were brought together, hence no particular region can be designated to a particular flow pattern in such a flow map. In exception of the combinations, (i) We/Eo versus $\mathrm{f}_{\mathrm{w}}$ and (ii) $\mathrm{Fr}_{\mathrm{m}}$ versus $\mathrm{f}_{\mathrm{w}}$, where $\mathrm{ST}, \mathrm{SM}, \mathrm{SL}$ and Dw/o were marked out.

- However, the flow patterns formed clearer trends on the flow map when the data points were regrouped, based on the oil-phase viscosity and pipe diameter used in the experimental studies.

- For regrouping based on oil viscosity, combinations of the ratio of mixture Reynold number to Eötvös number $\left(\operatorname{Re}_{m} / E o\right)$ versus water fraction $\left(f_{w}\right)$, and water Froude number $\left(\operatorname{Fr}_{w}\right)$ versus oil Froude number $\left(\mathrm{Fr}_{\mathrm{o}}\right)$ dimensionless groups marked out regions for all the considered flow patterns under the medium-viscous oil (MVO) category, though slug and annular flow patterns had only a few data points as compared to the other flow patterns.

- Under low-viscous oil (LVO) $<10$ group, there is no occurrence of annular flow, and hence the combination of these dimensionless groups $G / V$ versus $f_{w}$, and $F_{w}$ versus $F_{0}$ were able to normalise all the data points so that each flow pattern formed on a distinct region on the flow map.

- Under heavy-viscous oil (HVO) group, the ratio of mixture Reynold number to Eötvös number $\left(\mathrm{Re}_{\mathrm{m}} / \mathrm{Eo}\right)$ versus water fraction $\left(\mathrm{f}_{\mathrm{w}}\right)$ normalised all the data points except for the annular flow pattern. The ratio of gravity force to viscous force $(G / V)$ versus water fraction $\left(f_{W}\right)$ normalised all the data points except for dispersed oil in water and slug flow patterns, while the and water Froude number $\left(\mathrm{Fr}_{\mathrm{w}}\right)$ versus the oil Froude number $\left(\mathrm{Fr}_{\mathrm{o}}\right)$ could not normalise dispersed oil in water, slug and annular flow patterns.

- For the regrouping based on pipe diameters, as the range of considered pipe diameters narrows, the cluster of data points for each flow pattern forms a more distinct region on the flow map.

Author Contributions: Conceptualization, O.S.O. and G.F.; methodology, O.S.O., G.F. and L.L.; software, O.S.O.; validation, O.S.O.; formal analysis, O.S.O., G.F. and L.L.; investigation, O.S.O., G.F. and L.L.; resources, O.S.O., G.F. and L.L.; data curation, O.S.O.; writing-original draft preparation, O.S.O.; writing-review and editing, O.S.O., G.F., L.L. and A.E.; visualization, O.S.O., G.F., L.L. and A.E.; supervision, G.F. and L.L.; project administration, G.F. and L.L.; funding acquisition, G.F. and L.L. All authors have read and agreed to the published version of the manuscript.

Funding: The work has been carried out as part of EPSRC project MUltiphase Flow-induced Fluid-flexible structure InteractioN in Subsea applications (MUFFINS), EPSRC Reference EP/P033105/1. The Ph.D. studies of the corresponding author are sponsored by the Petroleum Technology Development Fund (PTDF), reference number PTDF/ED/PHD/OOS/1172/17.

Acknowledgments: The authors thank the partners of the EPSRC MUFFINS project, as well as those of the European Metrology Programme for Innovation and Research project (16ENG07-MultiFlowMet II), jointly funded by the European Commission and participating countries within Euramet and the European Union, for providing additional advice. Olusegun Samson Osundare, the corresponding author would like to thank the Petroleum Technology Development Fund (PTDF) for sponsoring his PhD studies.

Conflicts of Interest: The authors declare no conflict of interest. 


\section{Nomenclature}

Flow Pattern/ Abbreviation

3L

AN

AO

$\mathrm{Bb}$

CAF

D

DC

Do/w or Dow

Do/w \& w

Dw/o or Dwo

Dw/o \& Do/w \& w

Dw/o \& 0

Dw/o \& o/w

I

M

$O \& W / O$

o/w

PL

SL

SLw

SM

SMO

SMW

ST

ST \& MI

SW

SWD

w/o
Interpretation

Three layers flow

Annular/oil in water

concentric flow

Annular/oil annulus flow

Oil bubbles in water flow

Core-annular flow

Dispersed flow

Dual continuous flow

Dispersed oil in water flow

Dispersed oil in water with

water layer flow

Dispersed water in oil flow

Dispersed water in oil and

dispersed oil in water, and

water layer flow

Dispersed water in oil with oil ${ }_{\mathbf{g}}$

layer flow

Dispersed water in oil and

dispersed oil in water flow

Intermediate flow

Mixed flow

Oil layer and dispersed water

in oil flow

Oil-in-water emulsion flow

Plug flow

Oil slugs in water flow

Water slugs in oil flow

Stratified mixed flow

Stratified mixed/oil flow

Stratified mixed/water flow

Stratified flow

Stratified with mixing at

interface flow

Stratified wavy

Stratified wavy with droplet

at the interface (Stratified

wavy/drops) flow

Water-in-oil emulsion flow

\begin{tabular}{|c|c|c|}
\hline Symbol/ Abbreviation & Unit & Interpretation \\
\hline$\Delta \rho$ & - & Change in density \\
\hline A & $\mathrm{m}^{2}$ & Pipe cross-sectional area \\
\hline $\mathrm{d}$ & $\mathrm{m}$ & Diameter \\
\hline
\end{tabular}

d

DPG

Eo

Eo'

EOR

$\mathbf{f}_{\mathrm{f}}$

f

FPM

Fr

$\mathrm{g}$

G/V

HFI

HVO

LVO

MVO

$\mathrm{N}$

N/A

NP

OV

P

Q

$\boldsymbol{R e}_{\mathrm{m}} / \mathrm{Eo}$

RT

Subscript m

Subscript o

Subscript or

Subscript w

$\mathbf{v}_{\mathbf{m}}$

$\mathbf{v}_{\text {so }}$

$\mathbf{v}_{\text {sw }}$
$\mathrm{We}_{\mathrm{E}}$

Y

$\alpha$
$\theta$

$\theta$

$\rho$
Dimensionless parameter groups

Eötvös number

Modified Eötvös number

Enhanced oil recovery

Friction factor

Volume fraction

Flow pattern maps

Froude number

$\mathrm{m} / \mathrm{s}^{2} \quad$ Acceleration due to gravity

Ratio of the gravitational force to

viscous force

High-frequency impedance

Low-viscous oil

Medium-viscous oil

No, flow pattern not marked out

Not applicable

Not provided

Visual observation

Photography

$\mathrm{m}^{3} / \mathrm{s} \quad$ Volumetric flow rate

Ratio of mixture Reynolds

number to Eötvös number

Room temperature

Mixture

Oil phase

Organic phase

Water phase

Mixture velocity

Superficial oil velocity

Superficial water velocity

Weber number to Eötvös number

Yes, flow pattern marked out

Wettability

Inclination angle

$\mathrm{mPa}$ Oil viscosity

$\mathrm{Kg} / \mathrm{m}^{3} \quad$ Density

$\mathrm{N} / \mathrm{m} \quad$ Interfacial tension
Heavy-viscous oil

\section{References}

1. Angeli, P.; Hewitt, G.F. Pressure Gradient in Horizontal Liquid-Liquid Flows. Int. J. Multiph. Flow 1999, 24, 1183-1203. [CrossRef]

2. Brauner, N. Liquid-Liquid Two-Phase Flow Systems. In Modelling and Control of Two-Phase Phenomena; Bertola, V., Ed.; Springer: Vienna, Austria, 2003; pp. 221-279. [CrossRef]

3. Tan, J.; Jing, J.; Hu, H.; You, X. Experimental Study of the Factors Affecting the Flow Pattern Transition in Horizontal Oil-Water Flow. Exp. Therm. Fluid Sci. 2018, 98, 534-545. [CrossRef]

4. Brennen, C.E. Fundamentals of Multiphase Flow; Cambridge University Press: Cambridge, UK, 2005. 
5. Ibarra, R.; Zadrazil, I.; Markides, C.N.; Matar, O.K. Towards a Universal Dimensionless Map of Flow Regime. In Proceedings of the 11th International Conference on Heat Transfer, Fluid Mechanics and Thermodynamics, Skukuza, South Africa, 20-23 July 2015.

6. Russell, T.W.F.; Hodgson, G.W.; Govier, G.W. Horizontal Pipeline Flow of Mixtures of Oil and Water. Can. J. Chem. Eng. 1959, 37, 9-17. [CrossRef]

7. Charles, M.E.; Govier, G.W.; Hodgson, G.W. The Horizontal Pipeline Flow of Equal Density Oil-water Mixtures. Can. J. Chem. Eng. 1961, 39, 27-36. [CrossRef]

8. Charles, M.E.; Lilleleht, L.V. Correlation of Pressure Gradients for the Stratified Laminar-turbulent Pipeline Flow of Two Immiscible Liquids. Can. J. Chem. Eng. 1966, 44, 47-49. [CrossRef]

9. Valle, A. Multiphase Pipeline Flows in Hydrocarbon Recovery. Multiph. Sci. Technol. 1998, 10, 1-139. [CrossRef]

10. Ahmed, S.A.; John, B. Liquid-Liquid Horizontal Pipe Flow-A Review. J. Pet. Sci. Eng. 2018, 168, $426-447$. [CrossRef]

11. Trallero, J.L.; Sarica, C.; Brill, J.P. A Study of Oil/Water Flow Patterns in Horizontal Pipes. In SPE Annual Technical Conference and Exhibition; Society of Petroleum Engineers: Dallas, TX, USA, 1997; pp. 165-172. [CrossRef]

12. Al-Sarkhi, A.; Pereyra, E.; Mantilla, I.; Avila, C. Dimensionless Oil-Water Stratified to Non-Stratified Flow Pattern Transition. J. Pet. Sci. Eng. 2017, 151, 284-291. [CrossRef]

13. Paolinelli, L.D.; Rashedi, A.; Yao, J.; Singer, M. Study of Water Wetting and Water Layer Thickness in Oil-Water Flow in Horizontal Pipes with Different Wettability. Chem. Eng. Sci. 2018, 183, 200-214. [CrossRef]

14. Efird, K.D.; Jasinski, R.J. Effect of the Crude Oil on Corrosion of Steel in Crude Oil/Brine Production. Corrosion 1989, 45, 165-171. [CrossRef]

15. Cai, J.; Li, C.; Tang, X.; Ayello, F.; Richter, S.; Nesic, S. Experimental Study of Water Wetting in Oil-Water Two Phase Flow-Horizontal Flow of Model Oil. Chem. Eng. Sci. 2012, 73, 334-344. [CrossRef]

16. Shi, J.; Lao, L.; Yeung, H. Water-Lubricated Transport of High-Viscosity Oil in Horizontal Pipes: The Water Holdup and Pressure Gradient. Int. J. Multiph. Flow 2017, 96, 70-85. [CrossRef]

17. Arirachakaran, S.; Oglesby, K.D.; Malinowsky, M.S.; Shoham, O.; Brill, J.P. An Analysis of Oil/Water Flow Phenomena in Horizontal Pipes. In SPE Production \& Facilities; Society of Petroleum Engineers: Dallas, TX, USA, 1989.

18. Mukhaimer, A.; Al-sarkhi, A.; El Nakla, M.; Ahmed, W.H.; Al-hadhrami, L. Effect of Water Salinity on Flow Pattern and Pressure Drop in Oil-Water Flow. J. Pet. Sci. Eng. 2015, 128, 145-149. [CrossRef]

19. Piroozian, A.; Hemmati, M.; Ismail, I.; Manan, M.A.; Rashidi, M.M.; Mohsin, R. An Experimental Study of Flow Patterns Pertinent to Waxy Crude Oil-Water Two-Phase Flows. Chem. Eng. Sci. 2017, 164, 313-332. [CrossRef]

20. Oglesby, K.D. An Experimental Study on the Effect of Oil Viscosity, Mixture Velocity and Water Fraction on Horizontal Oil-Water Flow. Master's Thesis, The University of Tulsa, Tulsa, OK, USA, 1979.

21. Nädler, M.; Mewes, D. Flow Induced Emulsification in the Flow of Two Immiscible Liquids in Horizontal Pipes. Int. J. Multiph. Flow 1997, 23, 55-68. [CrossRef]

22. Al-Wahaibi, T.; Al-Wahaibi, Y.; Al-Ajmi, A.; Al-Hajri, R.; Yusuf, N.; Olawale, A.S.; Mohammed, I.A. Experimental Investigation on Flow Patterns and Pressure Gradient through Two Pipe Diameters in Horizontal Oil-Water Flows. J. Pet. Sci. Eng. 2014, 122, 266-273. [CrossRef]

23. Almutairi, Z.; Al-Alweet, F.M.; Alghamdi, Y.A.; Almisned, O.A.; Alothman, O.Y. Investigating the Characteristics of Two-Phase Flow Using Electrical Capacitance Tomography (ECT) for Three Pipe Orientations. Processes 2020, 8, 51. [CrossRef]

24. Cheng, L.; Ribatski, G.; Thome, J.R. Two-Phase Flow Patterns and Flow-Pattern Maps: Fundamentals and Applications. Appl. Mech. Rev. 2008, 61, 0508021-05080228. [CrossRef]

25. Torres, C.F.; Mohan, R.S.; Gomez, L.E.; Shoham, O. Oil-Water Flow Pattern Transition Prediction in Horizontal Pipes. J. Energy Resour. Technol. 2016, 138, 1-11. [CrossRef]

26. Hanafizadeh, P.; Hojati, A.; Karimi, A. Experimental Investigation of Oil-Water Two Phase Flow Regime in an Inclined Pipe. J. Pet. Sci. Eng. 2015, 136, 12-22. [CrossRef]

27. Cheng, H.; Hills, J.H.; Azzorpardi, B.J. A Study of the Bubble-to-Slug Transition in Vertical Gas-Liquid Flow in Columns of Different Diameter. Int. J. Multiph. Flow 1998, 24, 431-452. [CrossRef] 
28. Al-Wahaibi, T.; Yusuf, N.; Al-Wahaibi, Y.; Al-Ajmi, A. Experimental Study on the Transition between Stratified and Non-Stratified Horizontal Oil-Water Flow. Int. J. Multiph. Flow 2012, 38, 126-135. [CrossRef]

29. Yusuf, N.; Al-Wahaibi, Y.; Al-Wahaibi, T.; Al-Ajmi, A.; Olawale, A.S.; Mohammed, I.A. Effect of Oil Viscosity on the Flow Structure and Pressure Gradient in Horizontal Oil-Water Flow. Chem. Eng. Res. Des. 2012, 90, 1019-1030. [CrossRef]

30. Angeli, P.; Hewitt, G.F. Flow Structure in Horizontal Oil-Water Flow. Int. J. Multiph. Flow 2000, 26, 1117-1140. [CrossRef]

31. Huang, Z.; Lee, H.S.; Senra, M.; Scott Fogler, H. A Fundamental Model of Wax Deposition in Subsea Oil Pipelines. AIChE J. 2011, 57, 2955-2964. [CrossRef]

32. Tritton, D.J. Physical Fluid Dynamics; Springer: Dordrecht, The Netherlands, 1977. [CrossRef]

33. Hasson, D.; Mann, V.; Nir, A. Annular Flow of Two Immiscible Liquids I. Mechanisms. Can. J. Chem. Eng. 1970, 48, 514-520. [CrossRef]

34. Wegmann, A.; von Rohr, P.R. Two Phase Liquid-Liquid Flows in Pipes of Small Diameters. Int. J. Multiph. Flow 2006, 32, 1017-1028. [CrossRef]

35. Valle, A.; Utvik, O.H. Pressure Drop, Flow Pattern and Slip for Two-Phase Crude Oil/Water Flow: Experiments and Model Predictions. In International Symposium on Liquid-Liquid Two-Phase Flow and Transport Phenomena; Begellhouse: Danbury, CT, USA, 1997; pp. 1-12. [CrossRef]

36. Vedapuri, D.; Bessette, D.; Jepson, W.P. A Segregated Flow Model to Predict Water Layer Thickness in Oil-Water Flows in Horizontal and Slightly Inclined Pipelines. In Proceedings of the 8th International Conference Multiphase '97, Cannes, France, 18-20 June 1997; BHR Group, Ed.; Mechanical Engineering Publications: London, UK, 1997; pp. 75-106.

37. Simmons, M.J.H.; Azzopardi, B.J. Drop Size Distributions in Dispersed Liquid-Liquid Pipe Flow. Int. J. Multiph. Flow 2001, 27, 843-859. [CrossRef]

38. Guzhov, A.I.; Grishin, A.D.; Medredev, V.F.; Medredeva, O.P. Emulsion Formation during the Flow of Two Immiscible Liquids. Neft. Choz. 1973, 8, 58-61. (In Russian)

39. Brauner, N.; Moalem Maron, D. Flow Pattern Transitions in Two-Phase Liquid-Liquid Flow in Horizontal Tubes. Int. J. Multiph. Flow 1992, 18, 123-140. [CrossRef]

40. Ismail, A.S.I.; Ismail, I.; Zoveidavianpoor, M.; Mohsin, R.; Piroozian, A.; Sariman, M.Z.; Misnan, M.S. Review of Oil-Water through Pipes. Flow Meas. Instrum. J. 2015, 45, 357-374. [CrossRef]

41. Grassi, B.; Strazza, D.; Poesio, P. Experimental Validation of Theoretical Models in Two-Phase High-Viscosity Ratio Liquid-Liquid Flows in Horizontal and Slightly Inclined Pipes. Int. J. Multiph. Flow 2008, 34, 950-965. [CrossRef]

42. Bannwart, A.C.; Rodriguez, O.M.H.; de Carvalho, C.H.M.; Wang, I.S.; Vara, R.M.O. Flow Patterns in Heavy Crude Oil-Water Flow. J. Energy Resour. Technol. 2004, 126, 184. [CrossRef]

43. Bannwart, A.C. Wavespeed and Volumetric Fraction in Core Annular Flow. Int. J. Multiph. Flow 1998, 24, 961-974. [CrossRef]

44. Bordalo, S.N.; Oliveira, R.C. Experimental Study of Oil/ Water Flow With Paraffin Precipitation in Subsea Pipelines. In SPE Annual Technical Conference and Exhibition; Society of Petroleum Engineers: Dallas, TX, USA, 2007; pp. 1-8. [CrossRef]

45. Abubakar, A.; Al-Wahaibi, Y.; Al-Wahaibi, T.; Al-Hashmi, A.; Al-Ajmi, A.; Eshrati, M. Effect of Low Interfacial Tension on Flow Patterns, Pressure Gradients and Holdups of Medium-Viscosity Oil/Water Flow in Horizontal Pipe. Exp. Therm. Fluid Sci. 2015, 68, 58-67. [CrossRef]

46. Al-wahaibi, T.; Angeli, P. Experimental Study on Interfacial Waves in Stratified Horizontal Oil-Water Flow. Int. J. Multiph. Flow 2011, 37, 930-940. [CrossRef]

47. Wang, W.; Gong, J. Flow Regimes and Transition Characters of the High Viscosity Oil-Water Two Phase Flow. In Proceedings of the CPS/SPE International Oil \& Gas Conference and Exhibition, Beijing, China, 8-10 June 2010; pp. 1-8. [CrossRef]

48. Gafonova, O.V.; Yarranton, H.W. The Stabilization of Water-in-Hydrocarbon Emulsions by Asphaltenes and Resins. J. Colloid Interface Sci. 2001, 241, 469-478. [CrossRef]

49. Angeli, P. Liquid-Liquid Dispersed Flows in Horizontal Pipes. Ph.D. Thesis, Imperial College London, London, UK, 1996.

50. Trallero, J.L. Oil-Water Flow Patterns in Horizontal Pipes. Ph.D. Thesis, The University of Tulsa, Tulsa, OK, USA, 1995. 
51. Kurban, A.P.A.; Angeli, P.A.; Mendestatsis, M.A.; Hewitt, G.F. Stratified and Dispersed Oil-Water Flows in Horizontal Pipes. In Proceedings of the Multiphase 95, 7th International Conference, Cannes, France, 7-9 June 1995; pp. 277-291.

52. Soleimani, A. Phase Distribution and Associated Phenomena in Oil-Water Flows in Horizontal Tubes. Ph.D. Thesis, University of London, London, UK, 1999.

53. Mu, H. Experimental Research on Oil-Water Horizontal Pipe Flow. Master's Thesis, University of Petroleum, Beijing, China, 2001.

54. Xu, X.X. Study on Oil-Water Two-Phase Flow in Horizontal Pipelines. J. Pet. Sci. Eng. 2007, 59, 43-58. [CrossRef]

55. Lovick, J.; Angeli, P. Experimental Studies on the Dual Continuous Flow Pattern in Oil-Water Flows. Int. J. Multiph. Flow 2004, 30, 139-157. [CrossRef]

56. Chakrabarti, D.P.; Das, G.; Ray, S. Pressure Drop in Liquid-Liquid Two Phase Horizontal Flow: Experiment and Prediction. Chem. Eng. Technol. 2005, 28, 1003-1009. [CrossRef]

57. Vielma, M.; Atmaca, S.; Sarica, C.; Zhang, H.Q. Characterization of Oil/Water Flows in Horizontal Pipes. In SPE Annual Technical Conference and Exhibition; Society of Petroleum Engineers: Dallas, TX, USA, 2007. [CrossRef]

58. Dasari, A.; Desamala, A.B.; Dasmahapatra, A.K.; Mandal, T.K. Experimental Studies and Probabilistic Neural Network Prediction on Flow Pattern of Viscous Oil-Water Flow through a Circular Horizontal Pipe. Ind. Eng. Chem. Res. 2013, 52, 7975-7985. [CrossRef]

59. Voulgaropoulos, V.; Zhai, L.; Loannou, K.; Angeli, P. Evolution of Unstable Liquid-Liquid Dispersions in Horizontal Pipes. In Proceedings of the BHR Group-10th North American Conference on Multiphase Technology 2016, Banff, AB, Canada, 8-10 June 2016; pp. 305-318.

60. Hapanowicz, J. Phase Inversion in Liquid-Liquid Pipe Flow. Flow Meas. Instrum. 2010, 21, 284-291. [CrossRef]

61. Shi, J.; Yeung, H. Characterization of Liquid-Liquid Flows in Horizontal Pipes. AIChE J. 2017, 63, 1132-1143. [CrossRef]

62. Joseph, D.D.; Nguyen, K.; Beavers, G.S. Non-Uniqueness and Stability of the Configuration of Flow of Immiscible Fluids with Different Viscosities. J. Fluid Mech. 1984, 141, 319-345. [CrossRef]

63. Ibarra, R.; Markides, C.N.; Matar, O.K. A Review of Liquid-Liquid Flow Patterns in Horizontal and Slightly Inclined Pipes. Multiph. Sci. Technol. 2014, 26, 171-198. [CrossRef]

64. Mandal, T.K.; Chakrabarti, D.P.; Das, G. Oil Water Flow through Different Diameter Pipes: Similarities and Differences. Chem. Eng. Res. Des. 2007, 85, 1123-1128. [CrossRef]

65. Shoham, O. Mechanistic Modeling of Gas Liquid Two Phase Flow in Pipes; The Society of Petroleum Engineers (SPE): Tulsa, OK, USA, 2005.

66. Langhaar, H.L. Dimensional Analysis and Theory of Model; John Wiley \& Sons, Inc.: New York, NY, USA, 1951.

67. Morales-Ruiz, S.; Rigola, J.; Rodriguez, I.; Oliva, A. Numerical Resolution of the Liquid-Vapour Two-Phase Flow by Means of the Two-Fluid Model and a Pressure Based Method. Int. J. Multiph. Flow 2012, 43, 118-130. [CrossRef]

68. Levy, S. Two-Phase Flow in Complex Systems; John Wiley \& Sons: Hoboken, NJ, USA, 1999.

69. Dukler, A.E.; Wicks, M.; Cleveland, R.G. Frictional Pressure Drop in Two-phase Flow: B. An Approach through Similarity Analysis. AIChE J. 1964, 10, 44-51. [CrossRef]

(C) 2020 by the authors. Licensee MDPI, Basel, Switzerland. This article is an open access article distributed under the terms and conditions of the Creative Commons Attribution (CC BY) license (http://creativecommons.org/licenses/by/4.0/). 